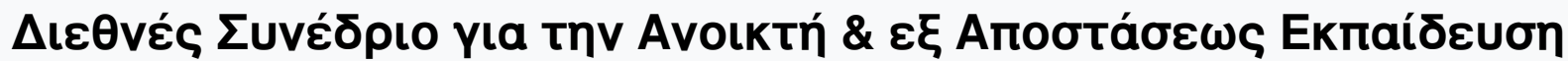

Tón. 6, Ap. 1A (2011)

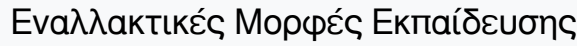

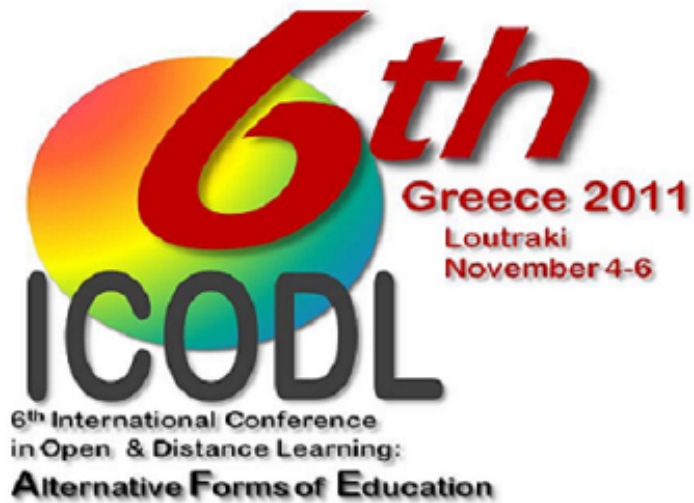

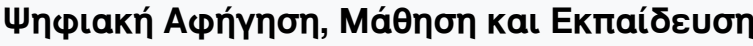

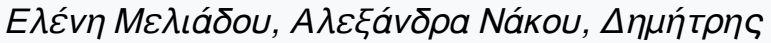

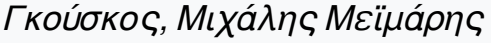

doi: $10.12681 /$ icodl. 681

TOMOEA

PART / MEPOEA 


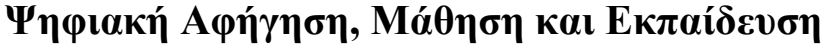

\section{Digital Storytelling, Learning and Education}

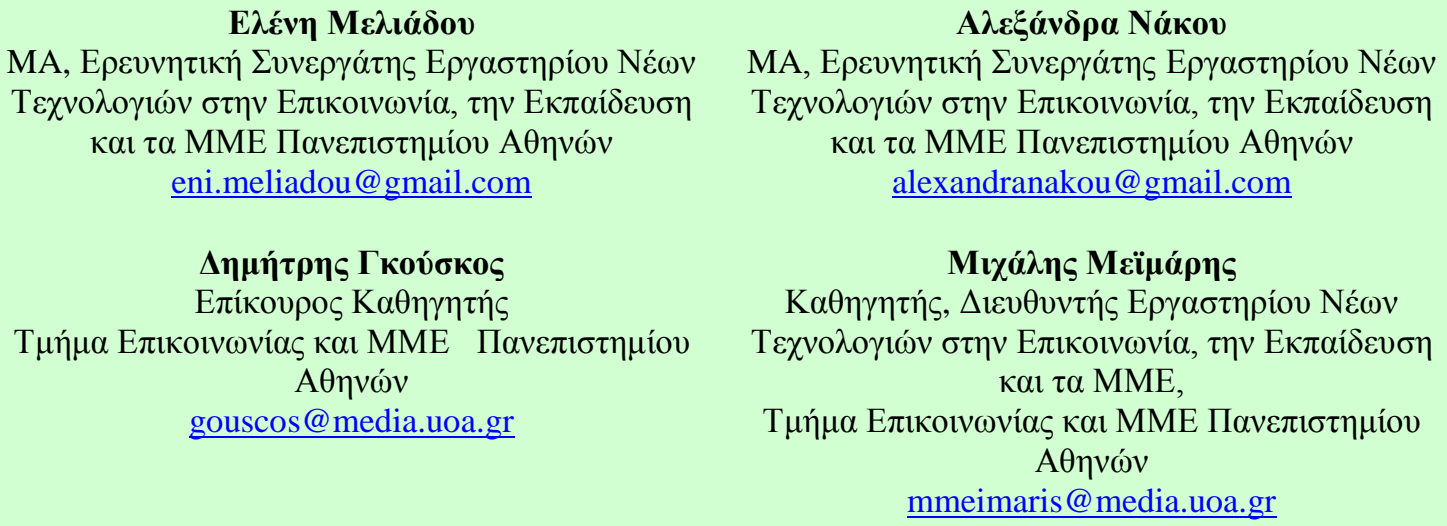

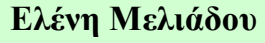

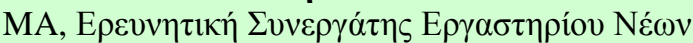

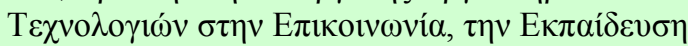

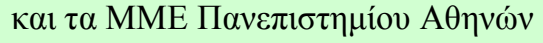
eni.meliadou@gmail.com

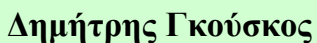

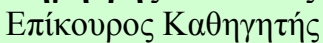

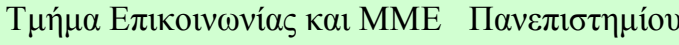
A $\theta$ ๆ gouscos@media.uoa.gr

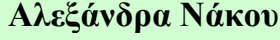

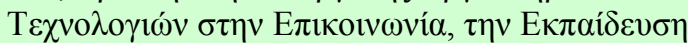

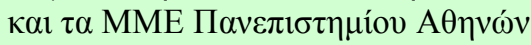 \\ alexandranakou@gmail.com
}

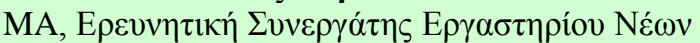

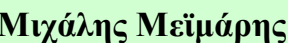

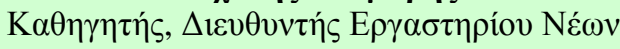

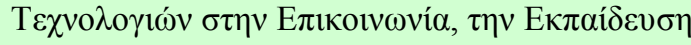
$\kappa \alpha 1 \tau \alpha \mathrm{MME}$,

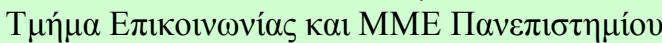
A $\theta \eta v \omega ́ v$

mmeimaris@media.uoa.gr

"...life must be lived forwards but it can only be understood backwards" Søren Kierkegaard

\begin{abstract}
Storytelling is present in all human activities throughout history. Part of the oral or written tradition storytelling, along with language, consist basic cultural procedures. Digital culture praises storytelling through different means of communication. Movies, the news, advertising, web pages present their content in a storytelling form. Digital storytelling is used in two different meanings, as an interactive experience, and also as a movement which defined digital storytelling as a narrated piece of personal writing, still images, video and musical soundtrack. Educational digital storytelling is offering new ways to communicate ideas and examples of goodpractices integrating digital storytelling in education are leading the way. The Laboratory of New Technologies in Communication, Education and the Mass Media acknowledges digital storytelling as an emerging field of study and focuses into research projects that develop from theory to practice. One of the accomplishments is the educational digital game "Magic Potion", where users enjoy the fun of playing along with participating in the reading of a story through their way. "Apple tree" is a storytelling platform where users can write, edit and share their stories on line, another is a workshop titled "Stars and producers of digital memories" combining digital storytelling and intergenerational learning
\end{abstract}

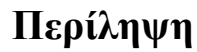

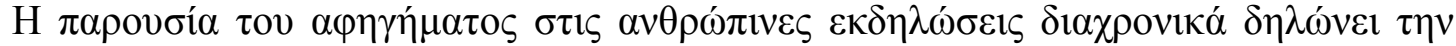

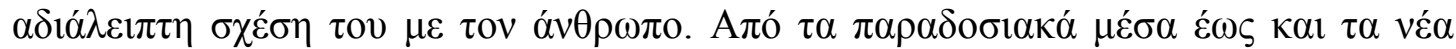

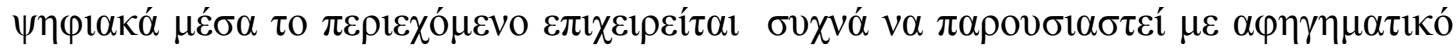

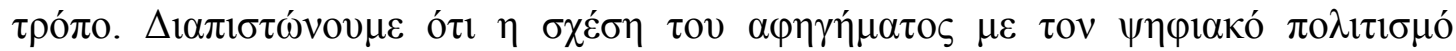

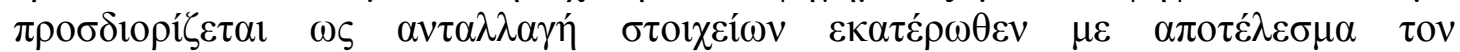

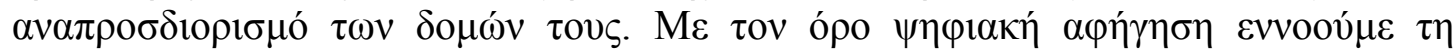

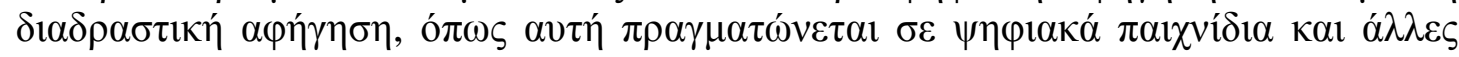

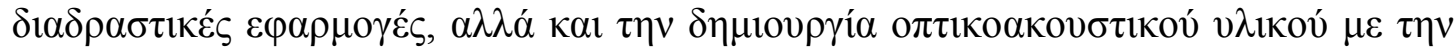




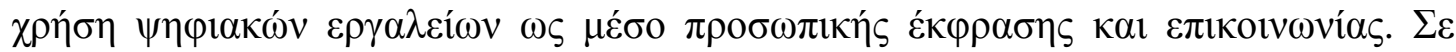

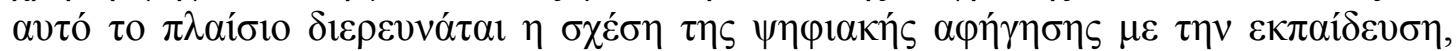

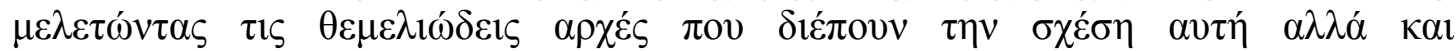

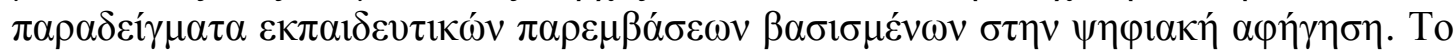

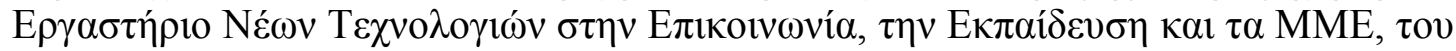

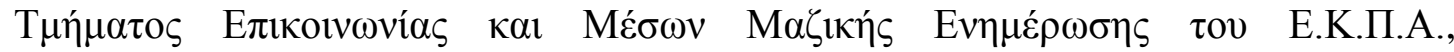

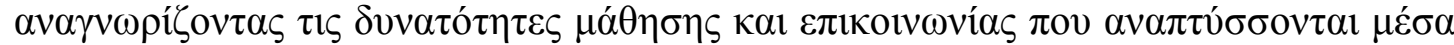

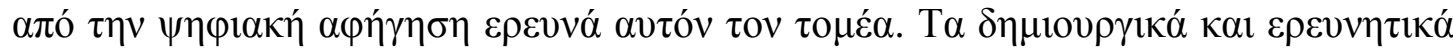

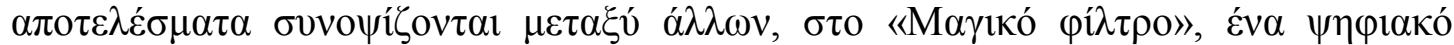

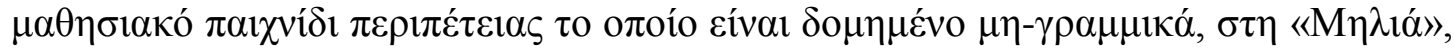

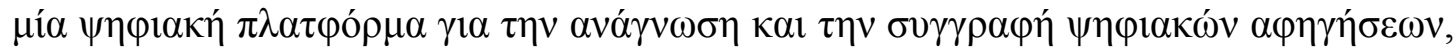

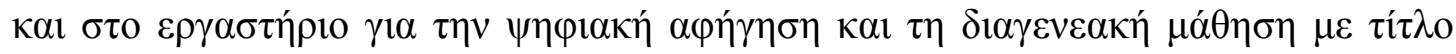

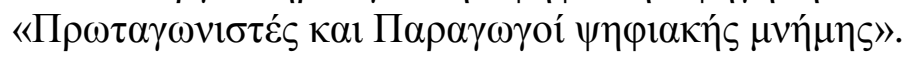

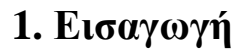

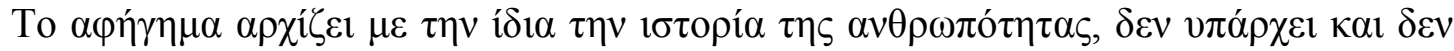

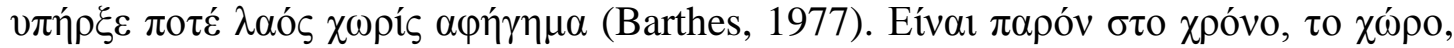

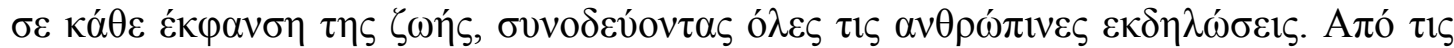

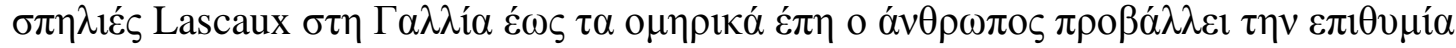

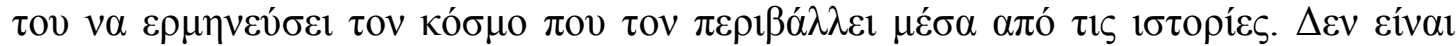

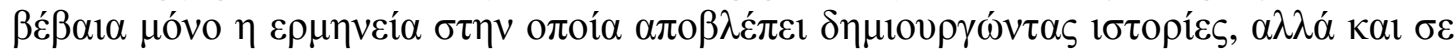

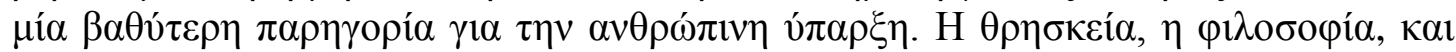

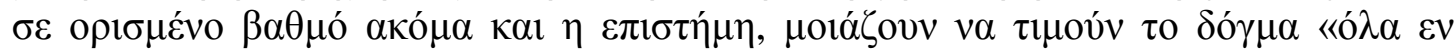

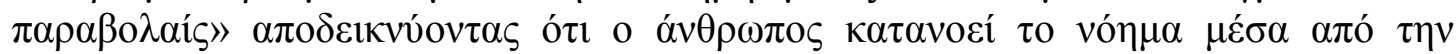

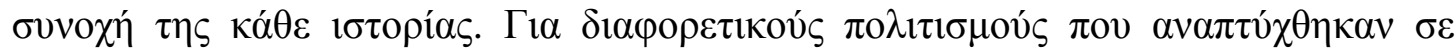

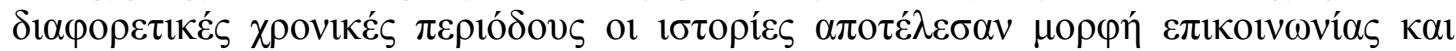

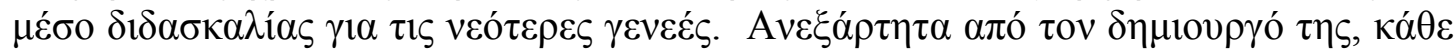

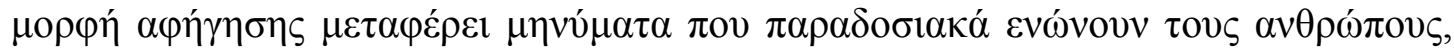

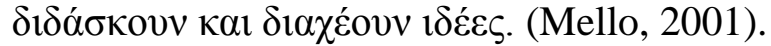

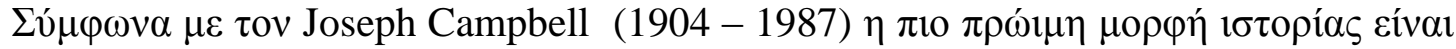

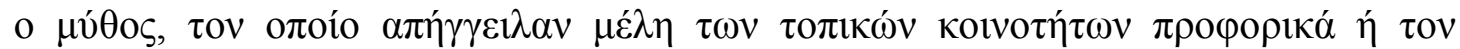

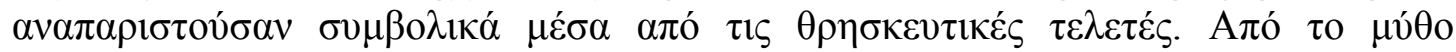

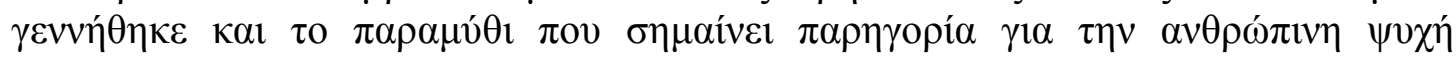

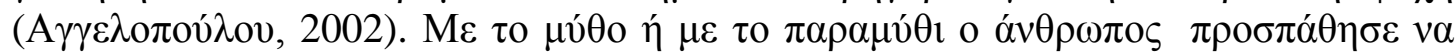

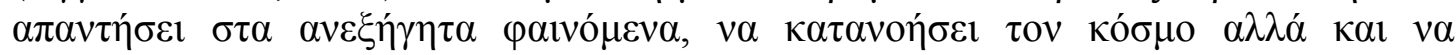

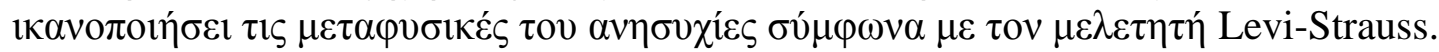

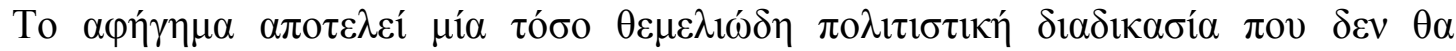

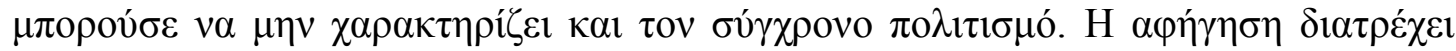

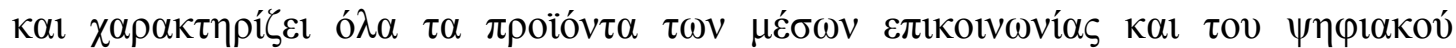

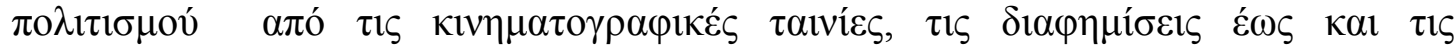

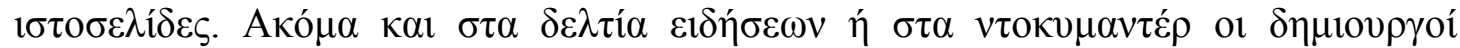

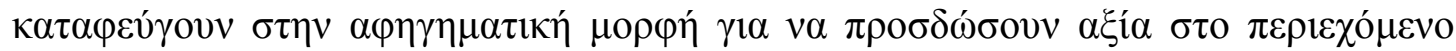

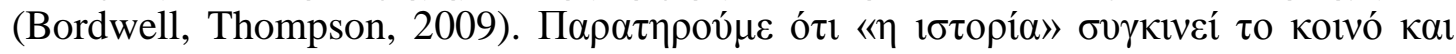

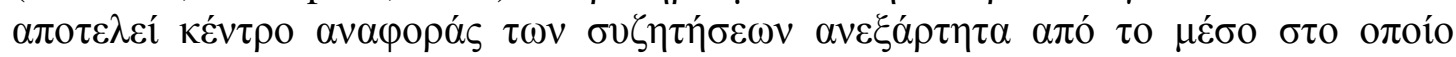

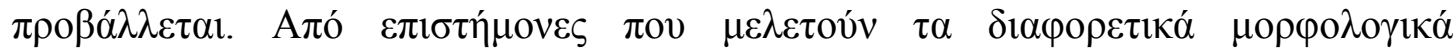

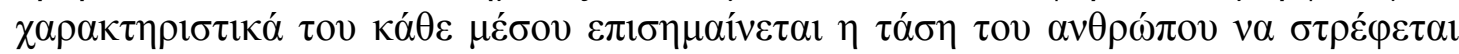

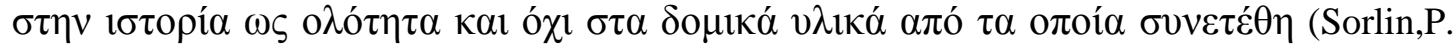

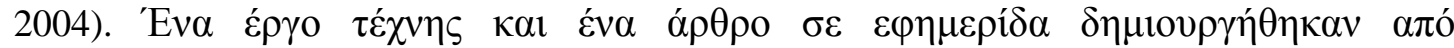

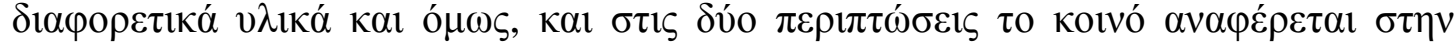

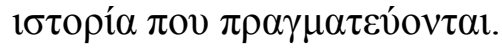




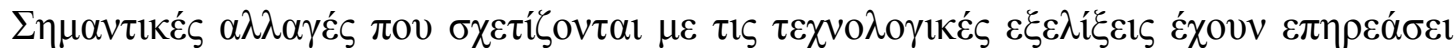

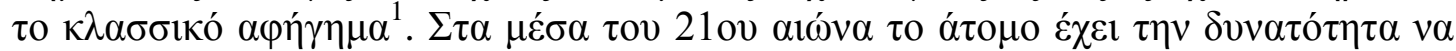

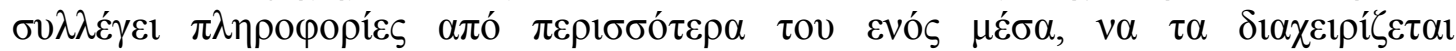

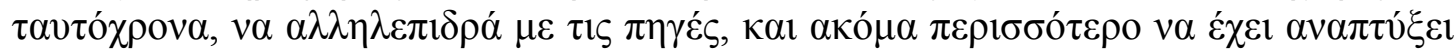

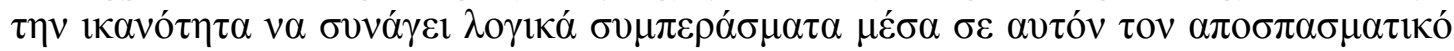

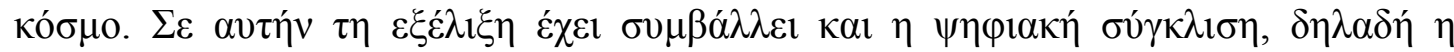

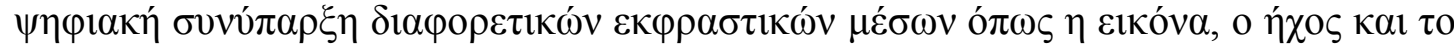

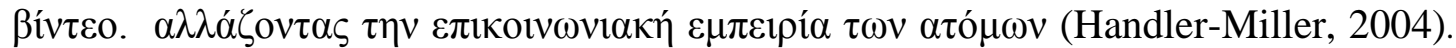

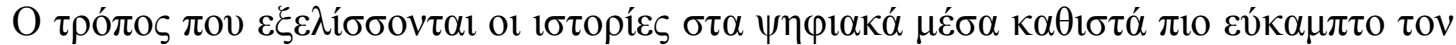

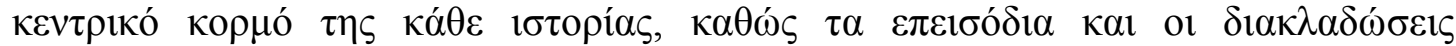

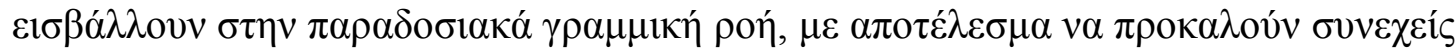

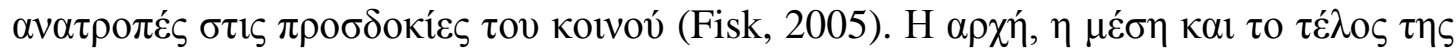

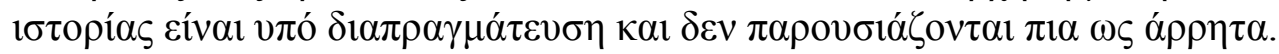

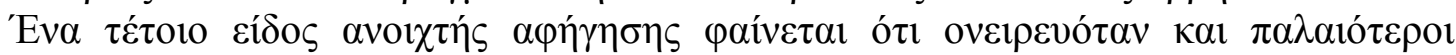

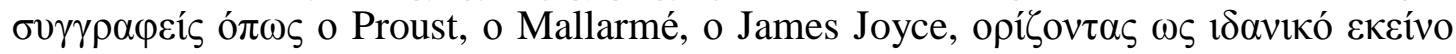

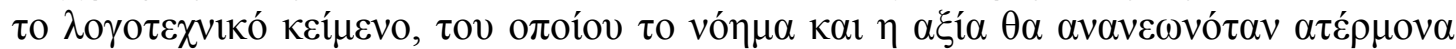

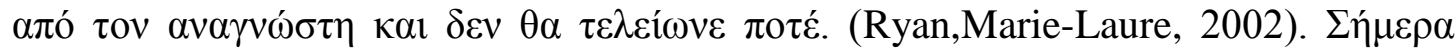

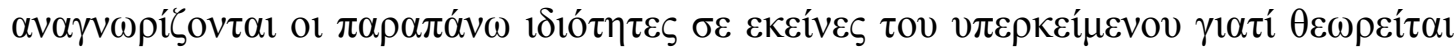

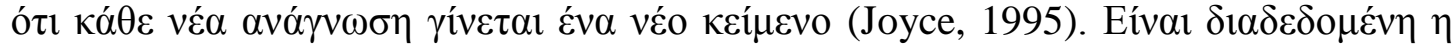

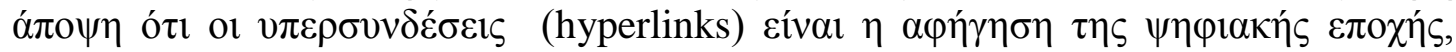

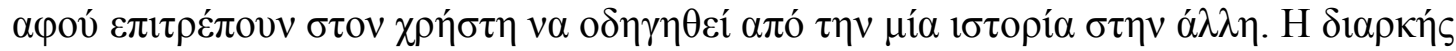

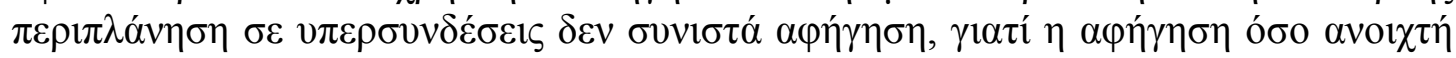

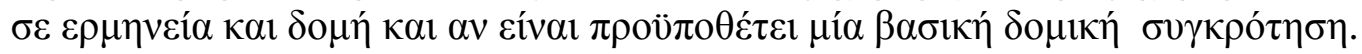

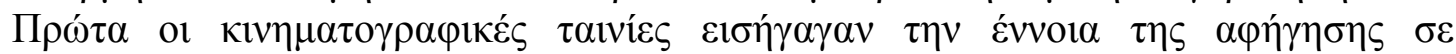

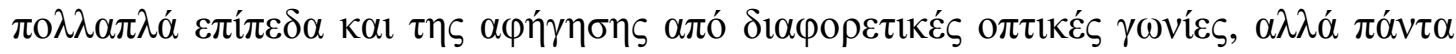

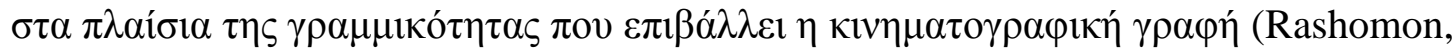

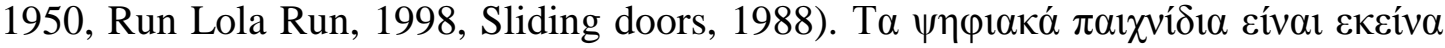

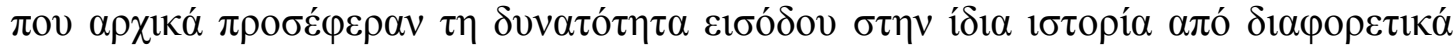

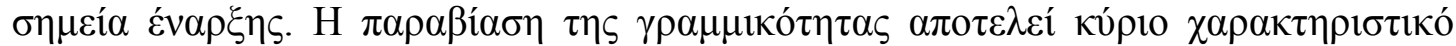

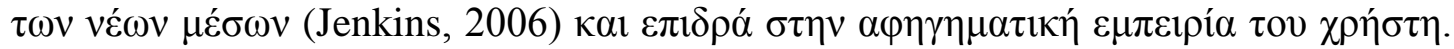

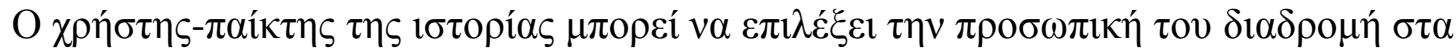

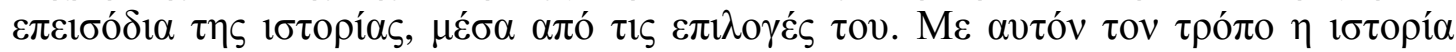

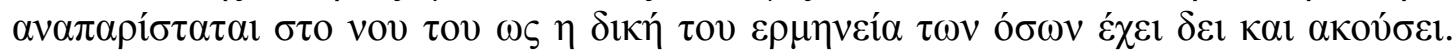

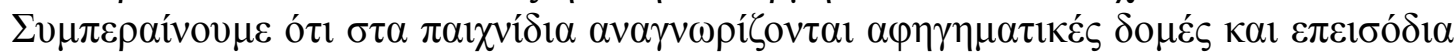

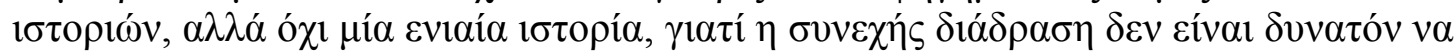

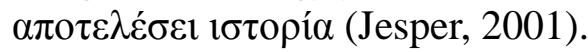

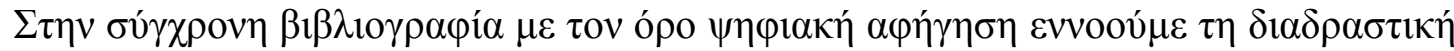

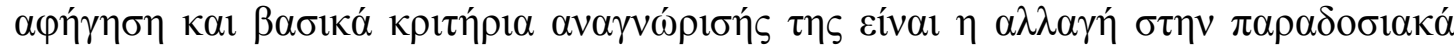

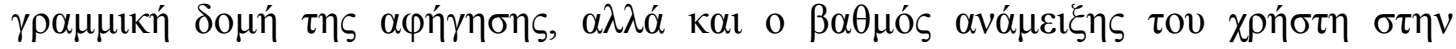

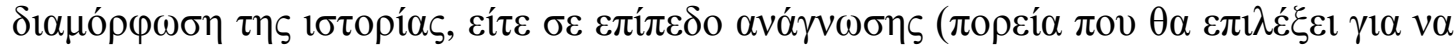

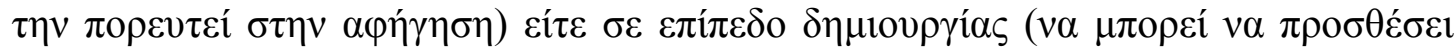

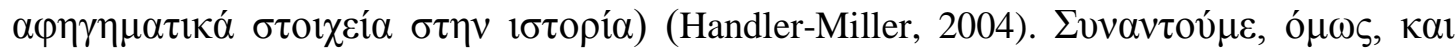

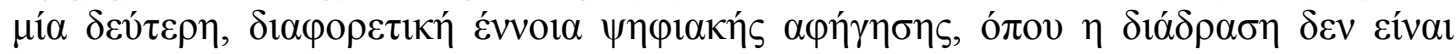

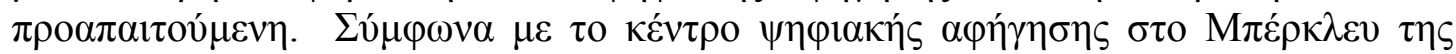

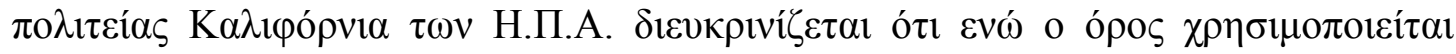

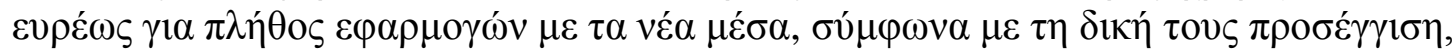

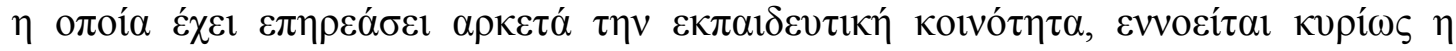

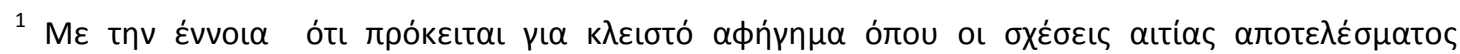

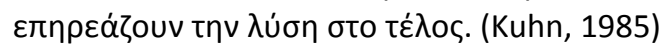




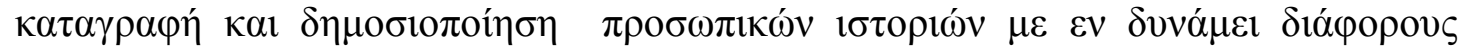
бколоús. (www.storycenter.org).

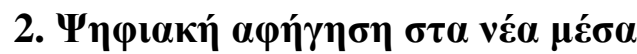

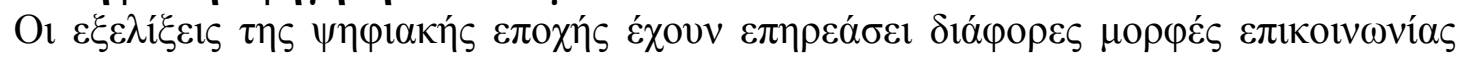

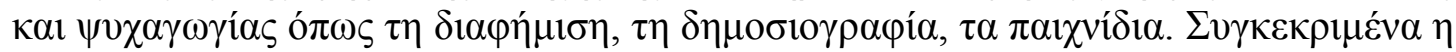

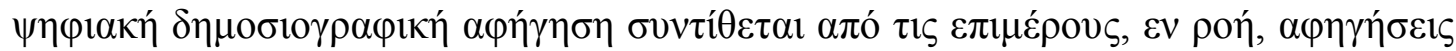

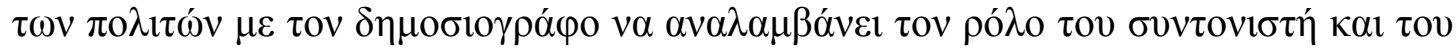

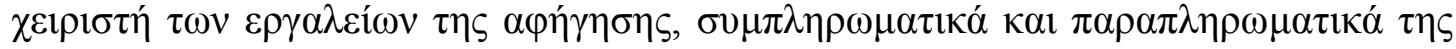

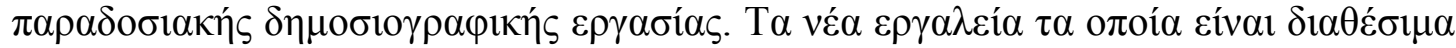

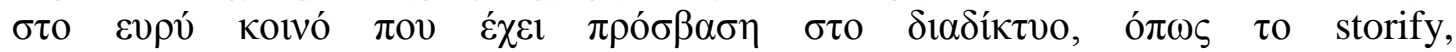

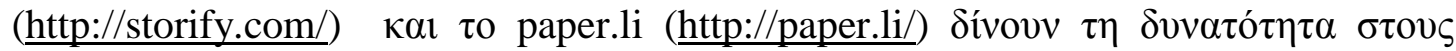

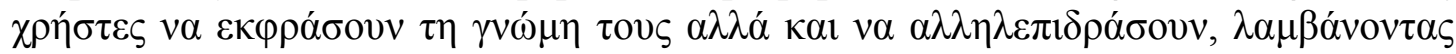

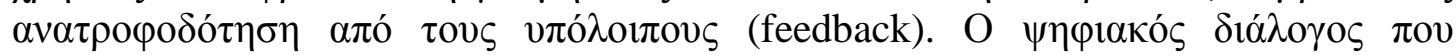

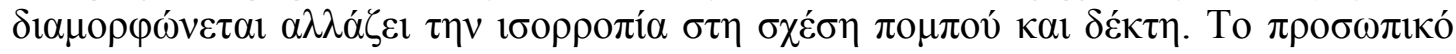

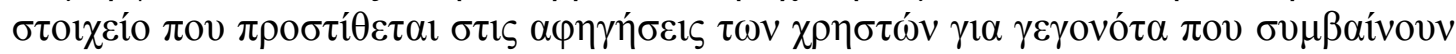

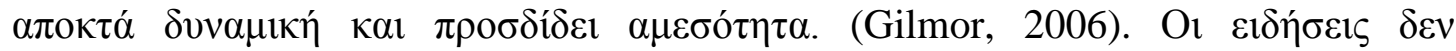

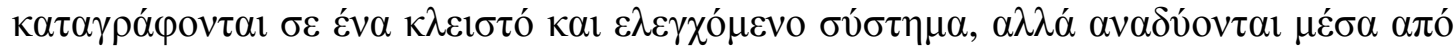

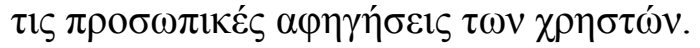

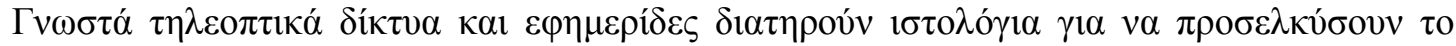

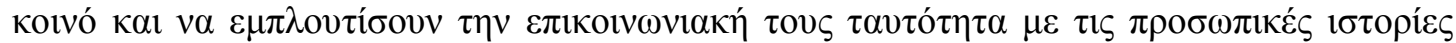

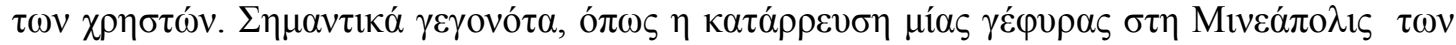

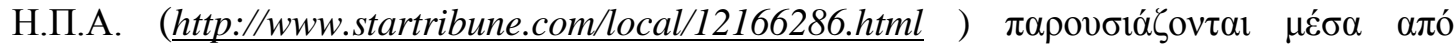

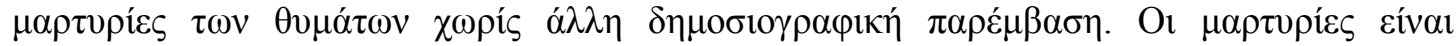

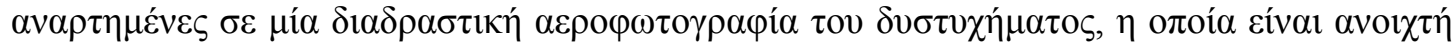

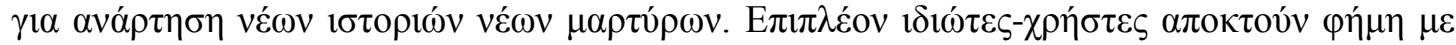

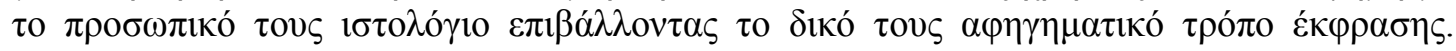

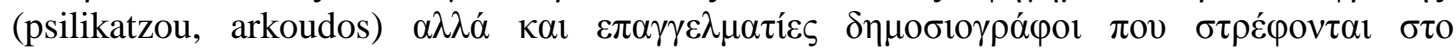

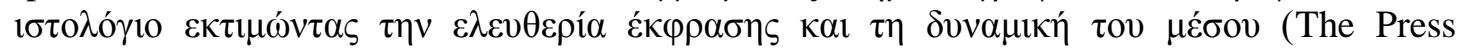
Project, pitsirikos)

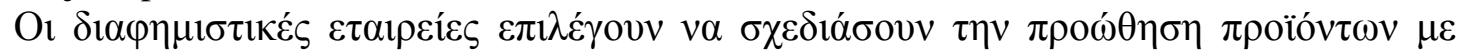

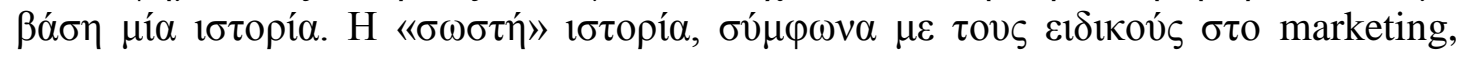

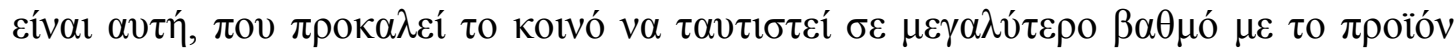

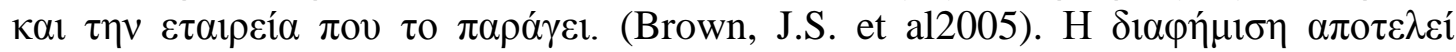

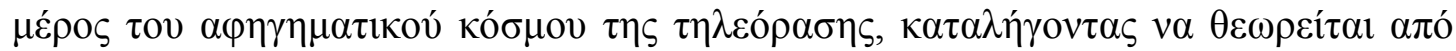

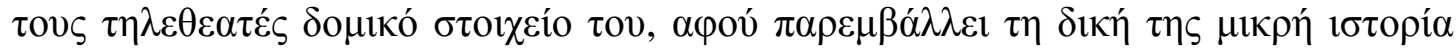

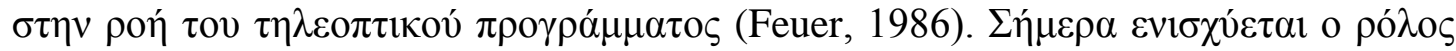

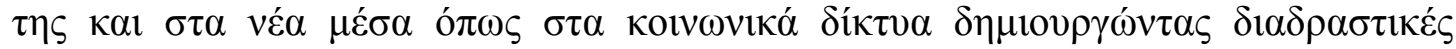

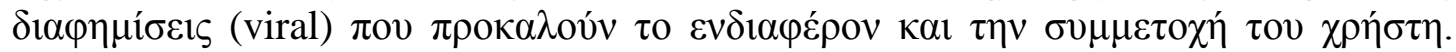

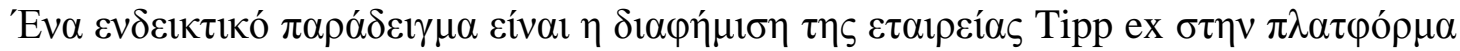

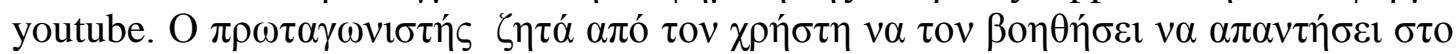

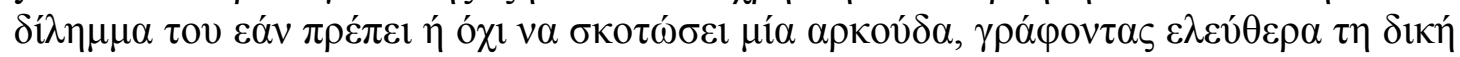

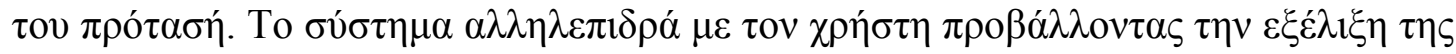

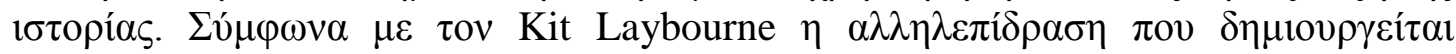

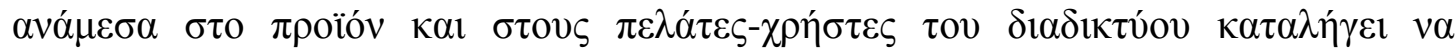

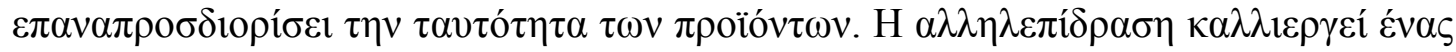

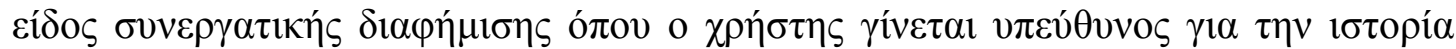

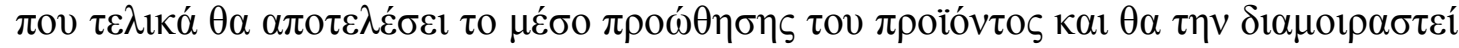

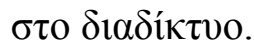

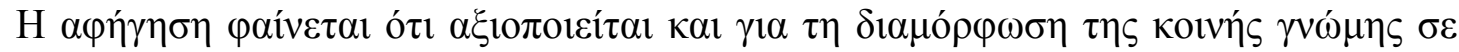

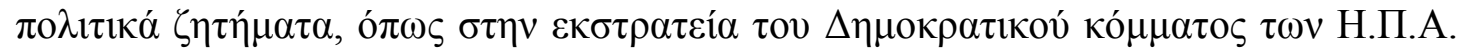




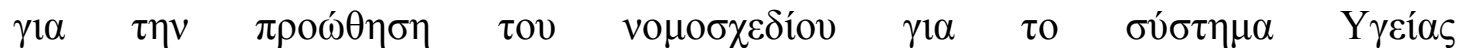

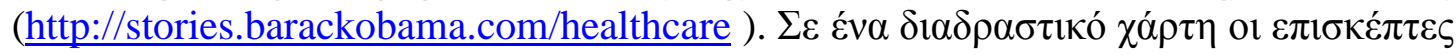

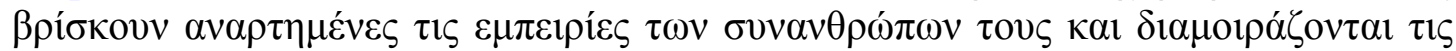

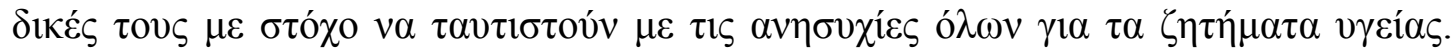

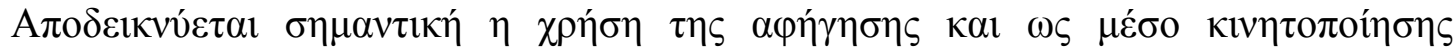

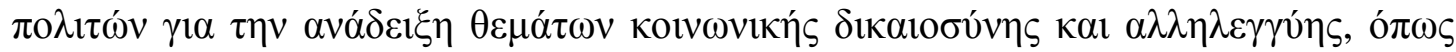
$\pi \alpha \rho \alpha \tau \eta \rho о v ́ \mu \varepsilon \sigma \tau \eta v ~ \imath \sigma \tau о \sigma \varepsilon \lambda i ́ \delta \alpha$ Silence Speaks (http://www.silencespeaks.org/ ). Or

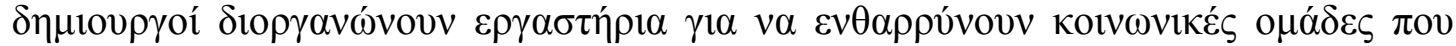

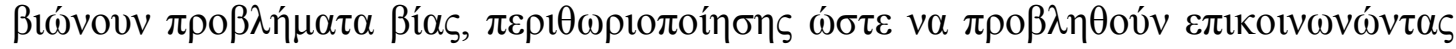

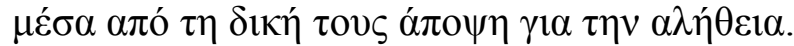

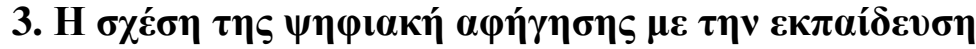

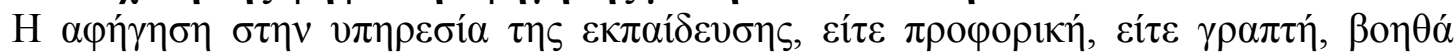

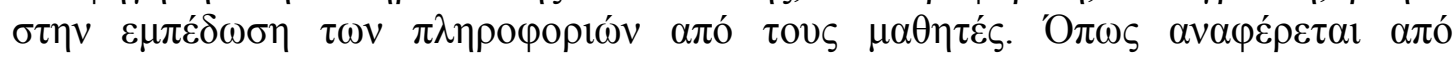

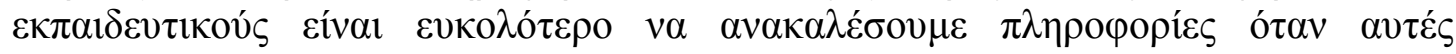

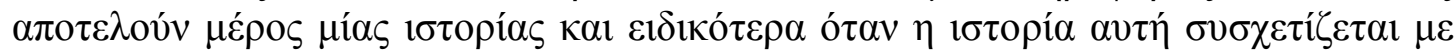

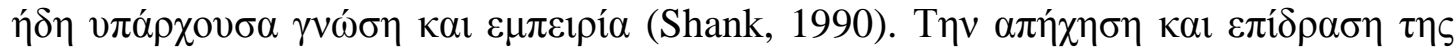

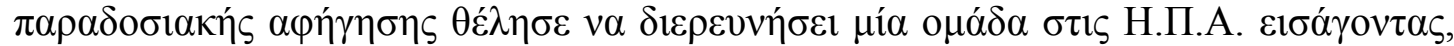

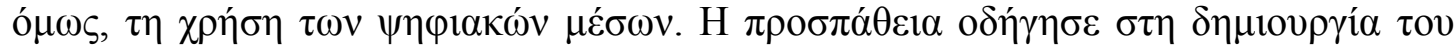

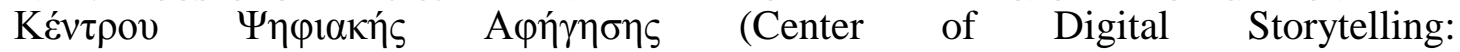

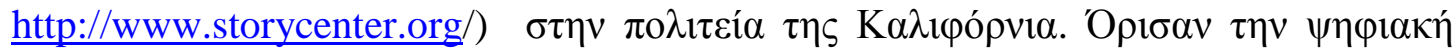

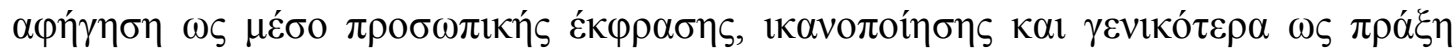

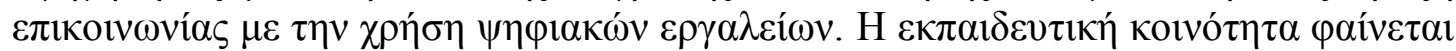

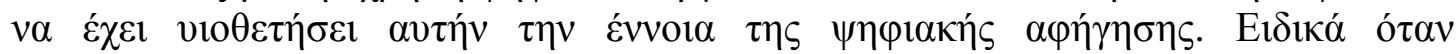

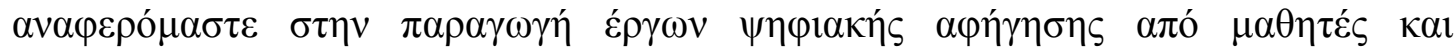

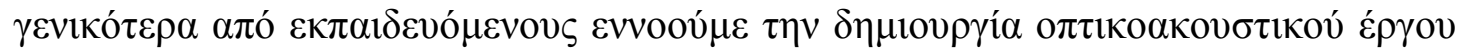

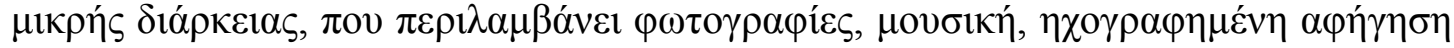

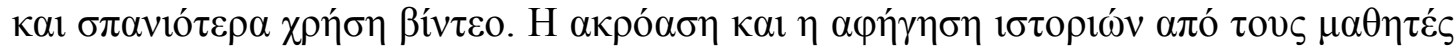

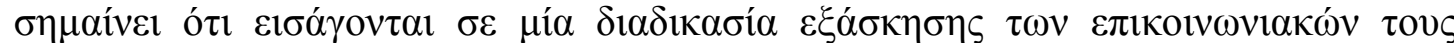

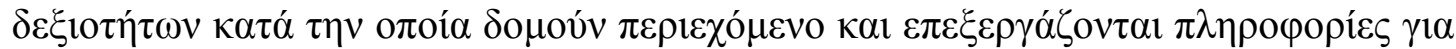
$v \alpha \kappa \alpha \tau \alpha \sigma \kappa \varepsilon v \alpha ́ \sigma o v v$ vó $\mu \alpha$

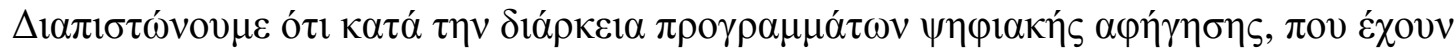

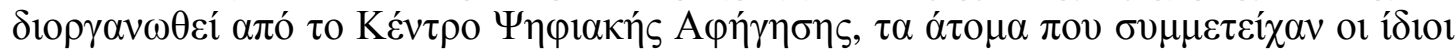

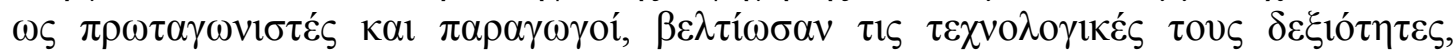

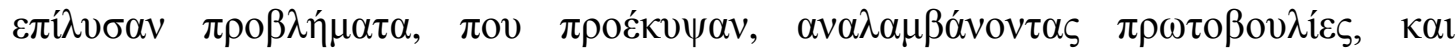

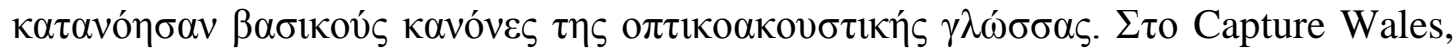

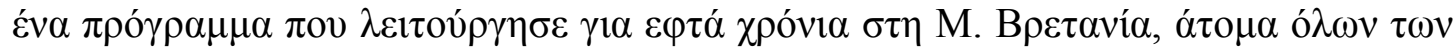

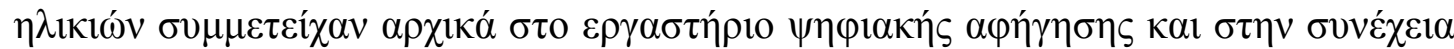

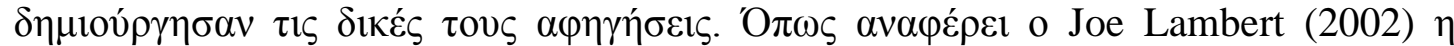

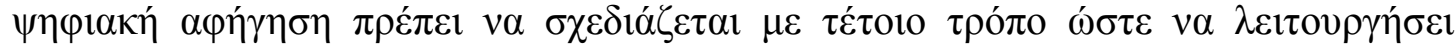

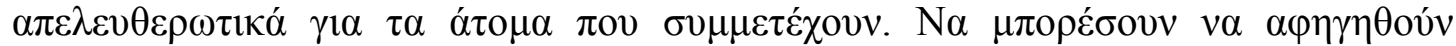

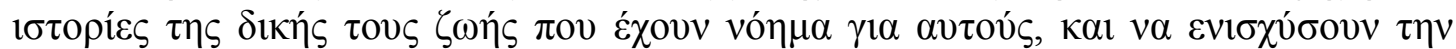

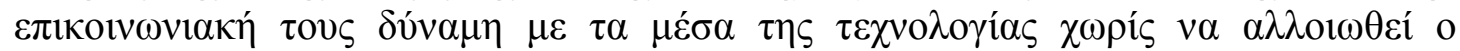

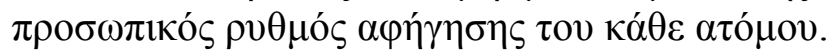

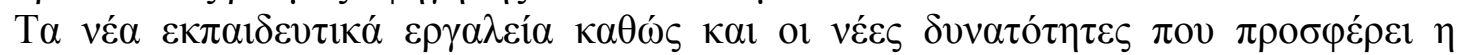

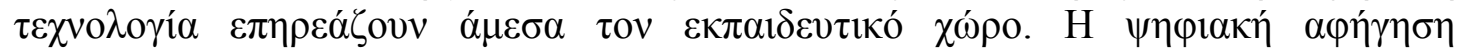

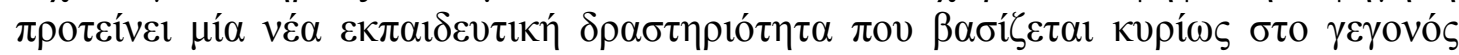

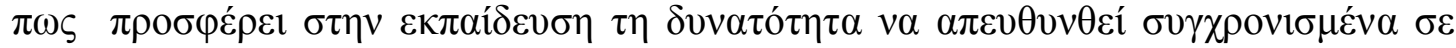

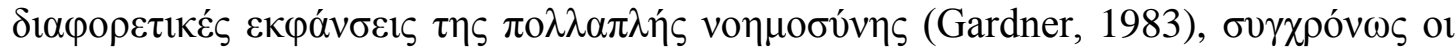




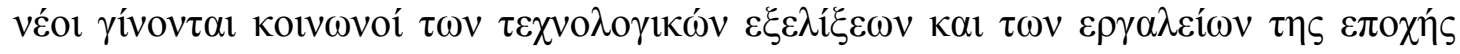
tovs.

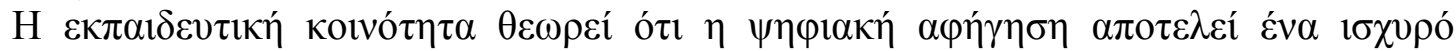

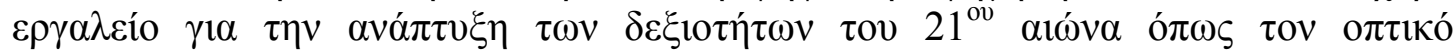

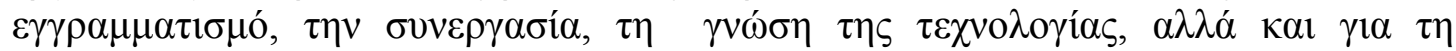

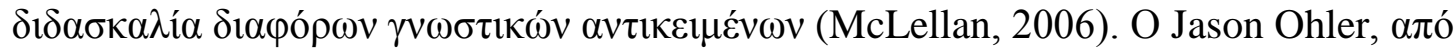

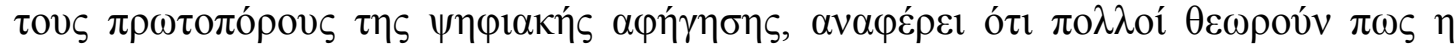

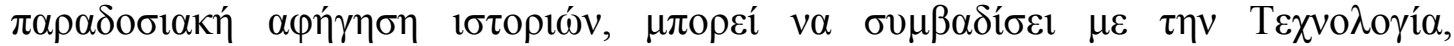

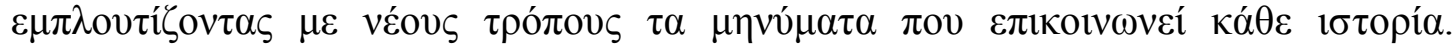

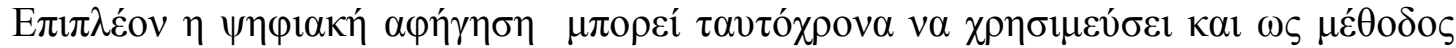

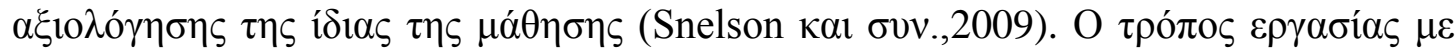

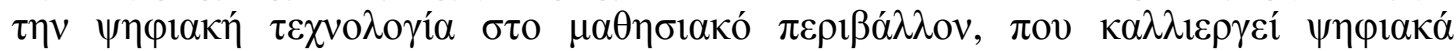

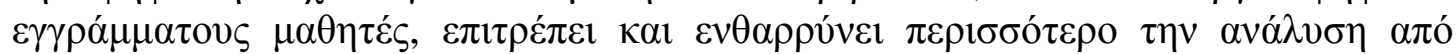

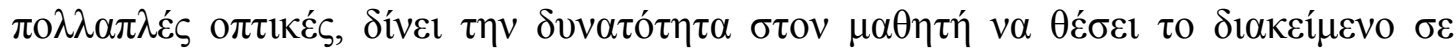

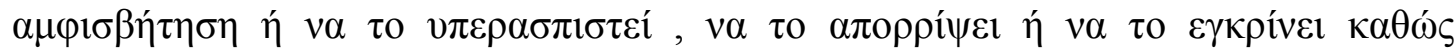

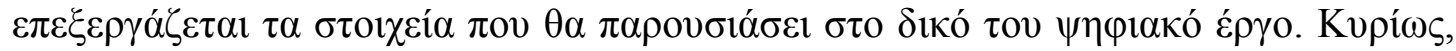

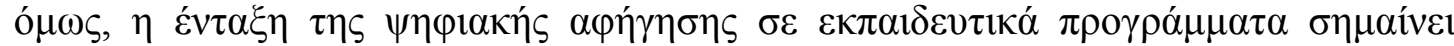

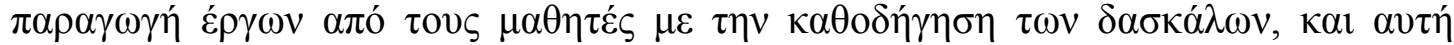

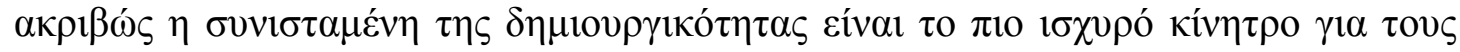
$\mu \alpha \theta \eta \tau \varepsilon ́ c$. (Frazel, 2010)

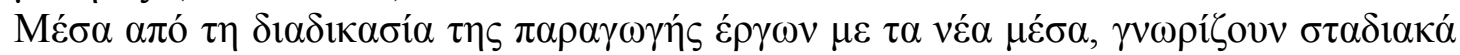

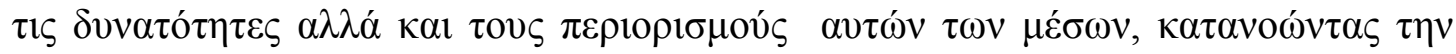
$\alpha \lambda \lambda \eta \lambda \varepsilon \xi \dot{\alpha} \rho \tau \eta \sigma \eta \delta$

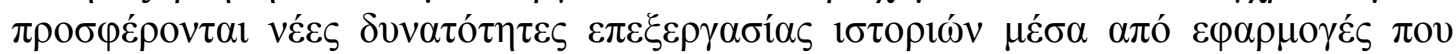

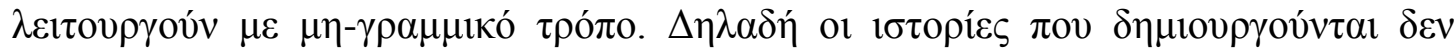

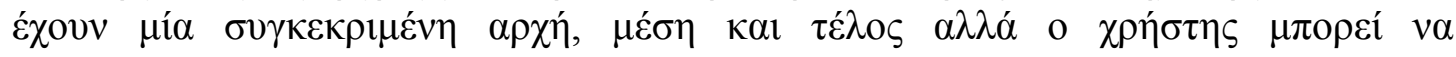

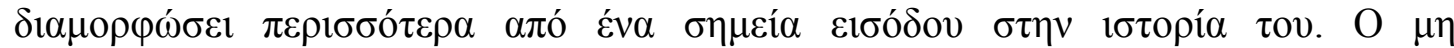

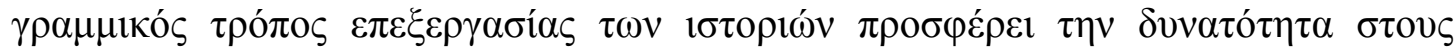

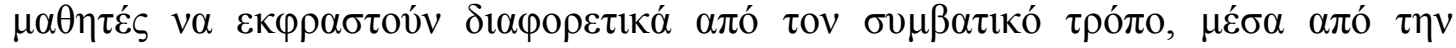

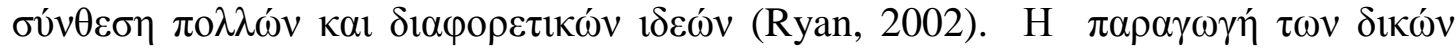

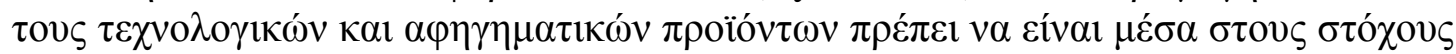

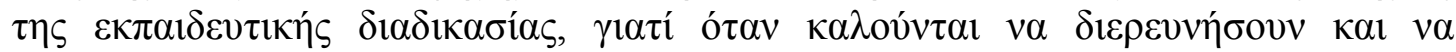

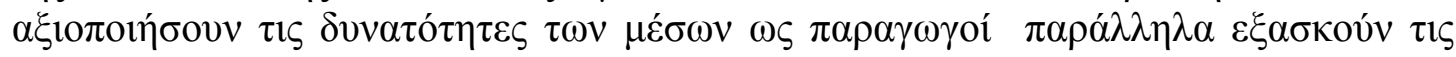

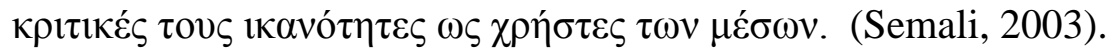

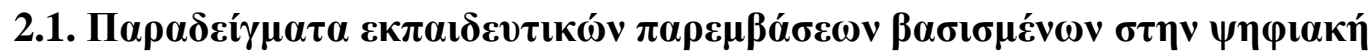 $\alpha \varphi \eta ́ \gamma \eta \sigma \eta$}

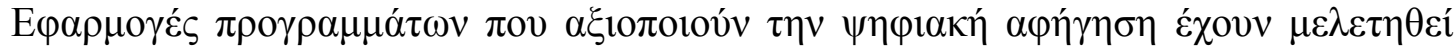

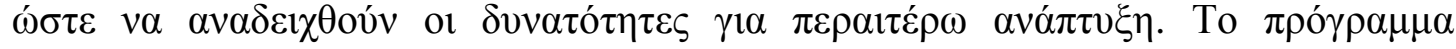

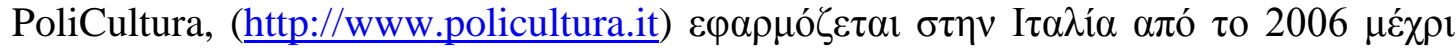

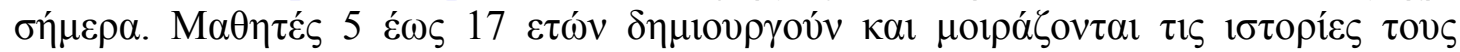

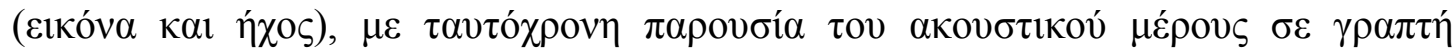

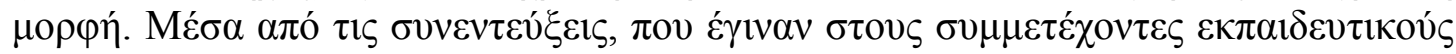

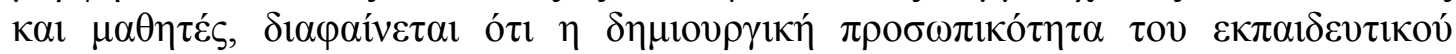

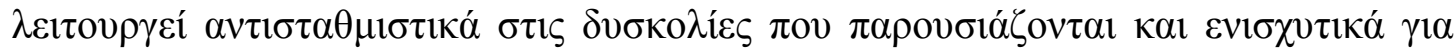

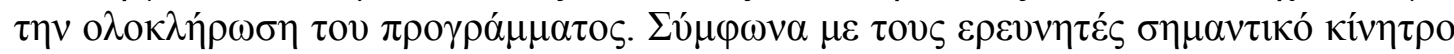

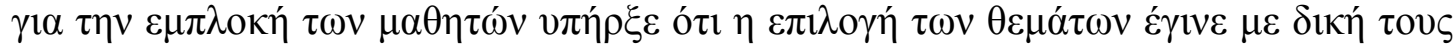

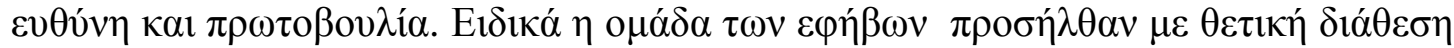

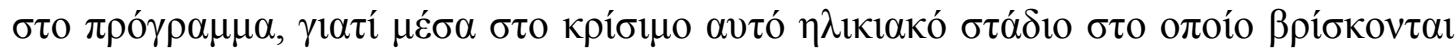

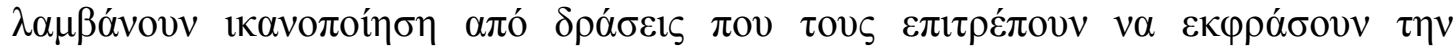




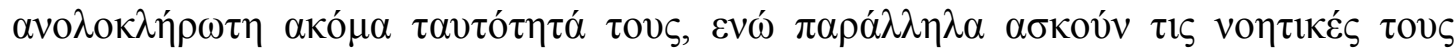

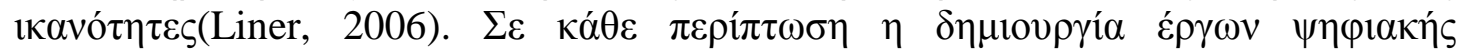

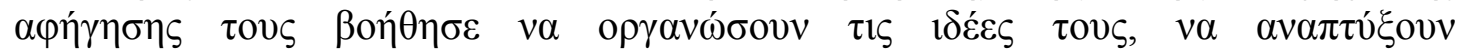

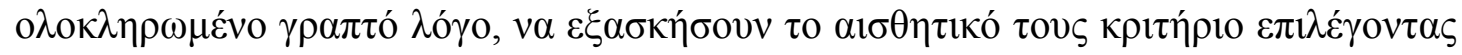

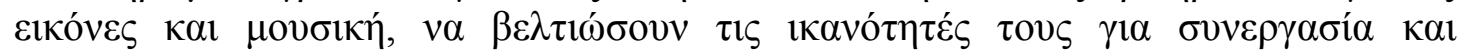

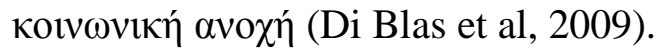

$\Sigma \tau \alpha$ a

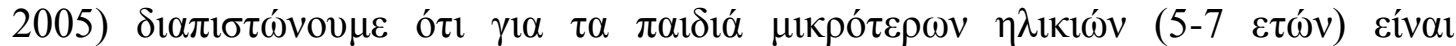

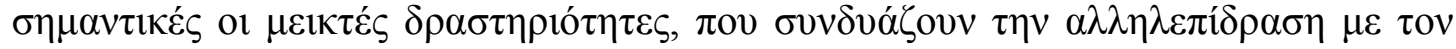

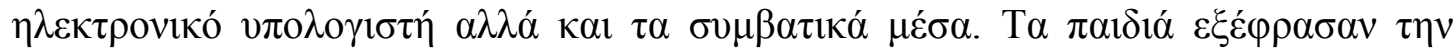

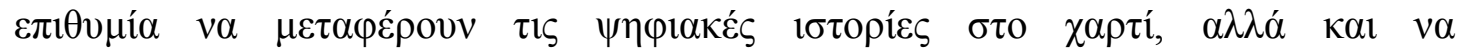

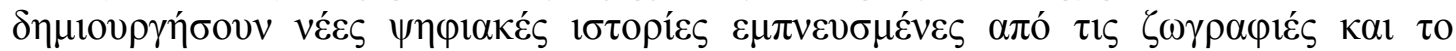

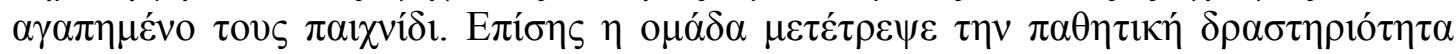

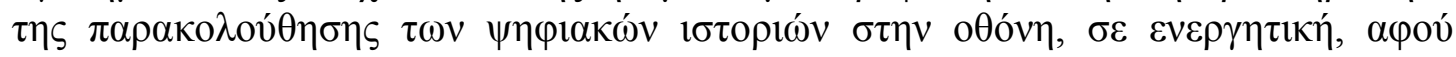

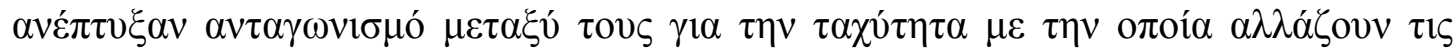

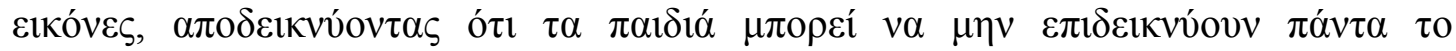

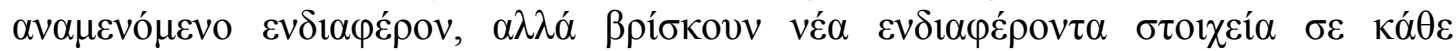

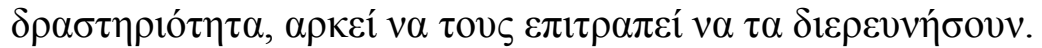

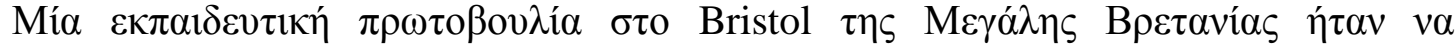

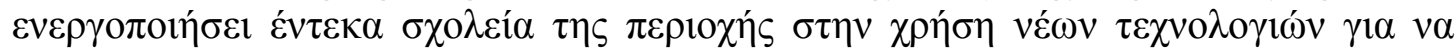

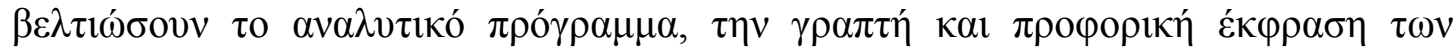

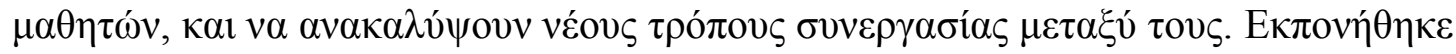

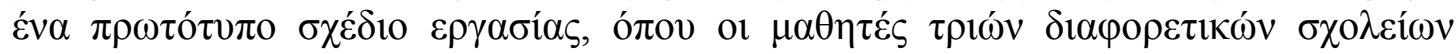

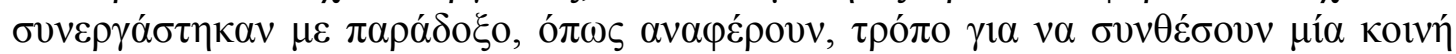

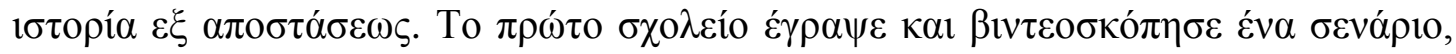

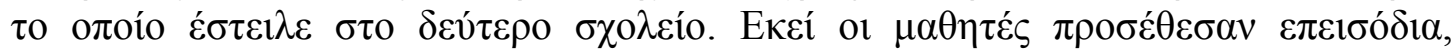

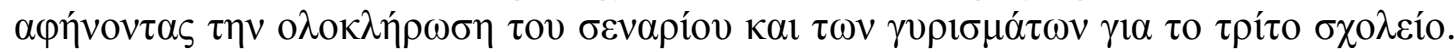

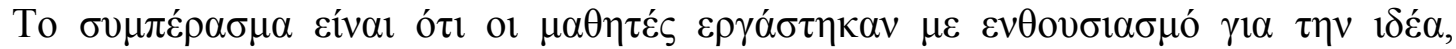

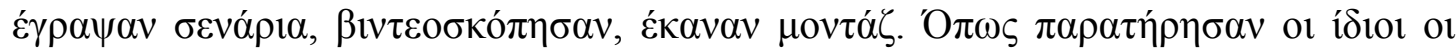

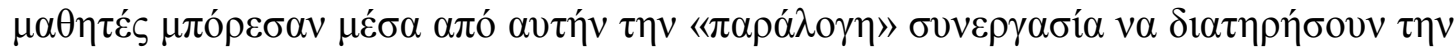

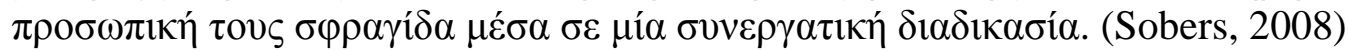

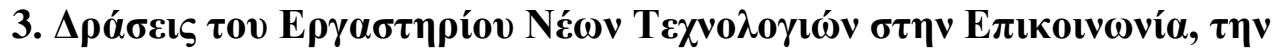

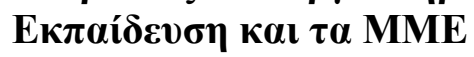

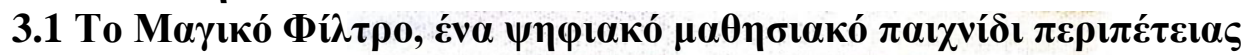

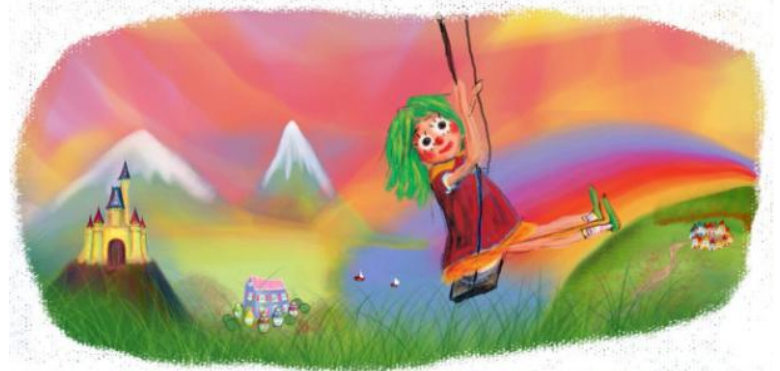

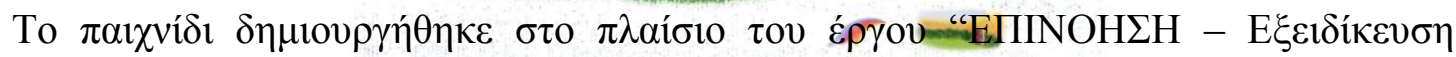

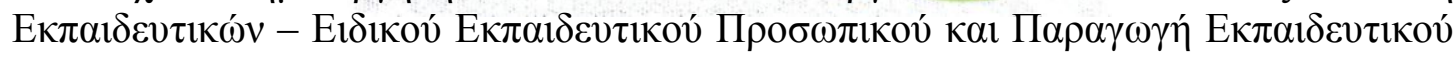

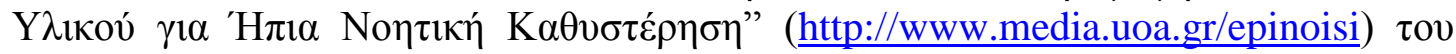

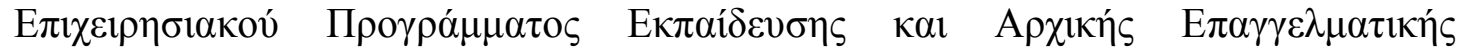

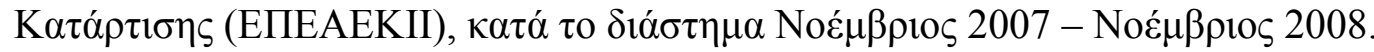

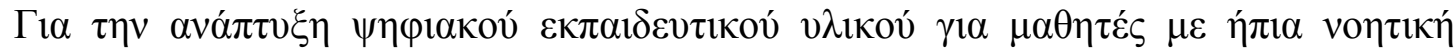

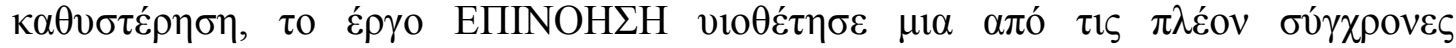




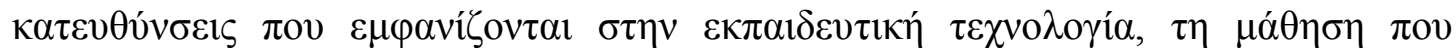

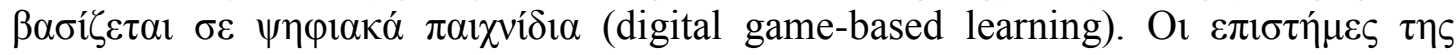

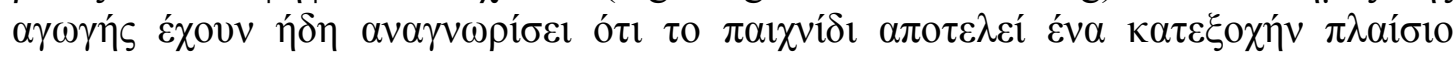

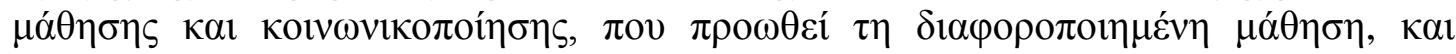

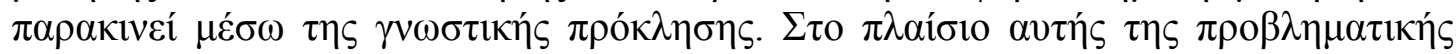

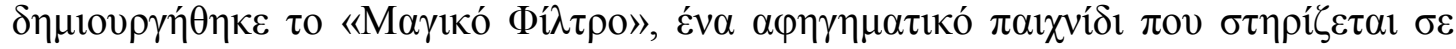

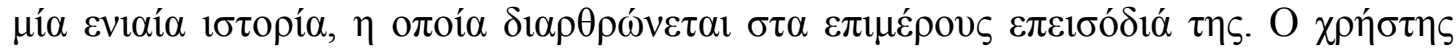

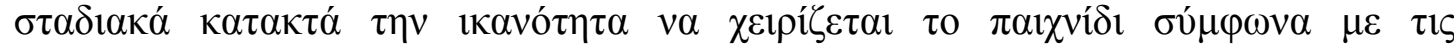

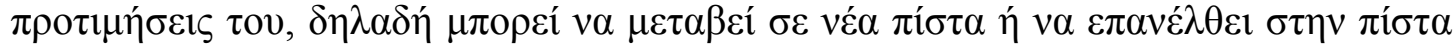
$\pi \mathrm{ov} \pi \rho \mathrm{\rho} \tau \mu \alpha \dot{\alpha}$.

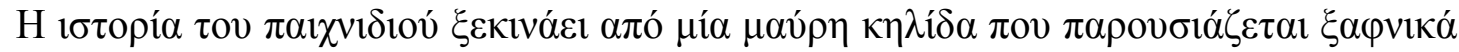

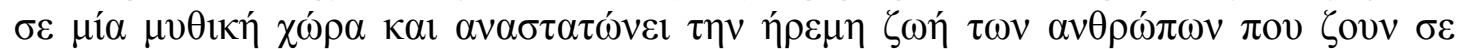

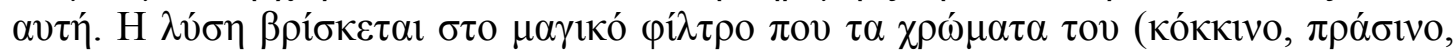

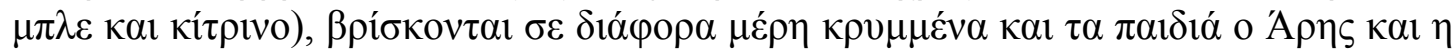

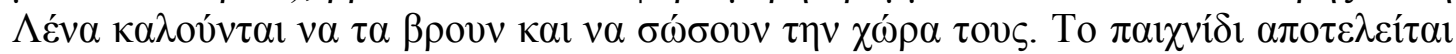

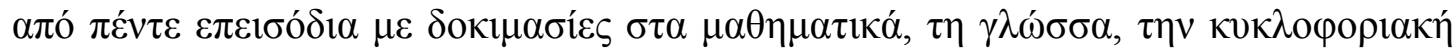

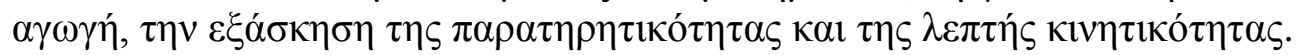

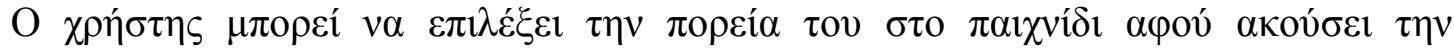

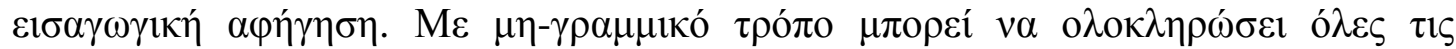

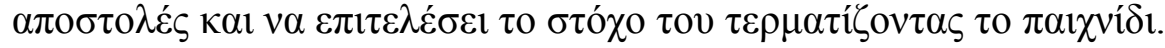

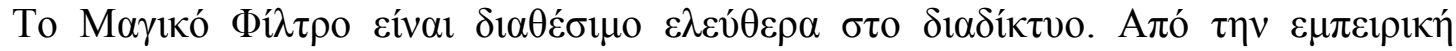

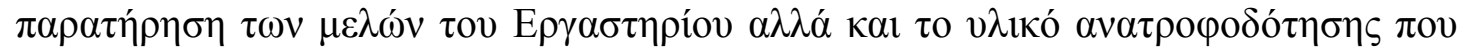

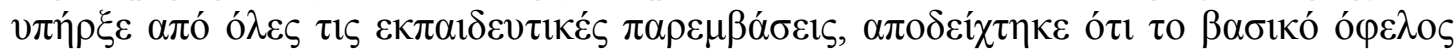

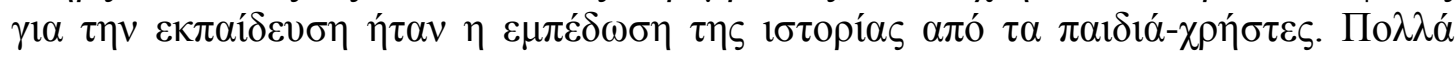

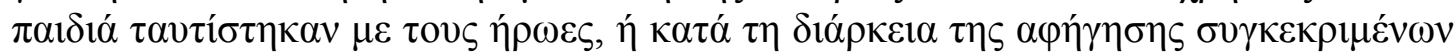

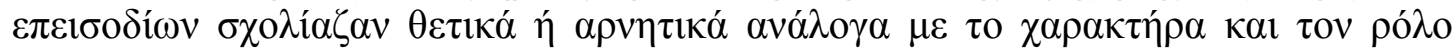

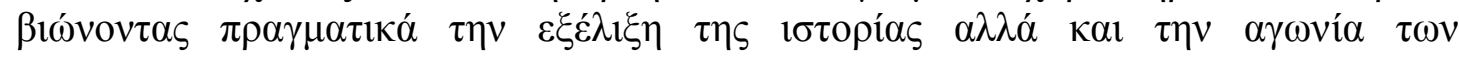

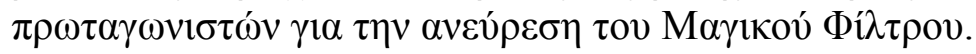

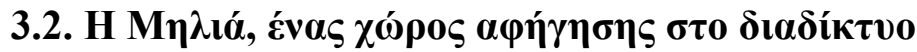




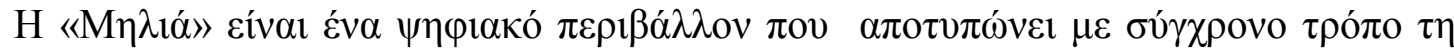

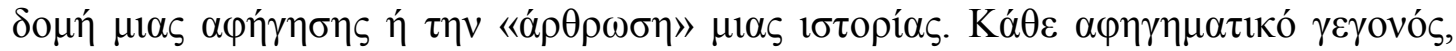

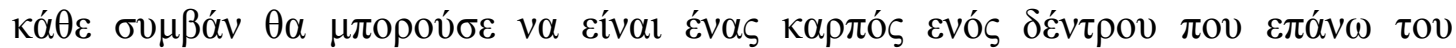

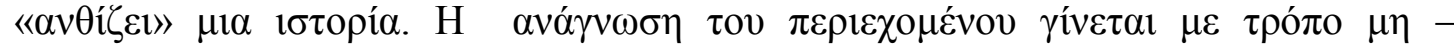

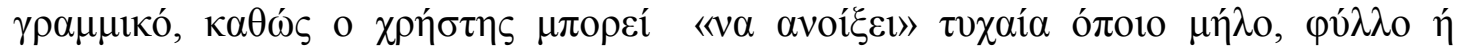

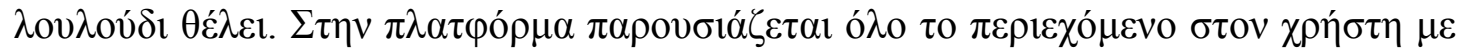

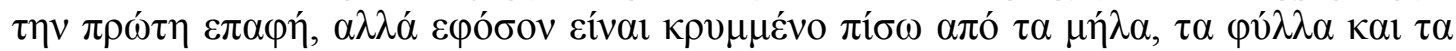

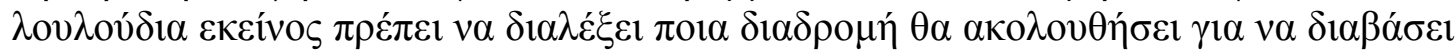

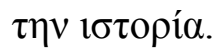

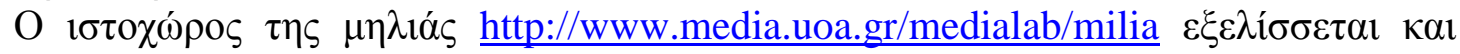

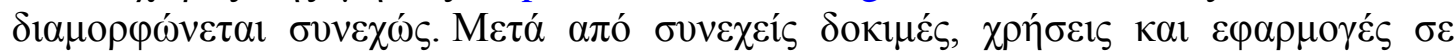

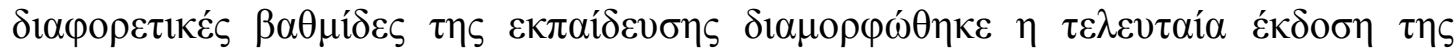

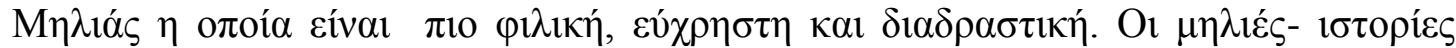

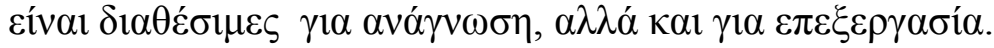

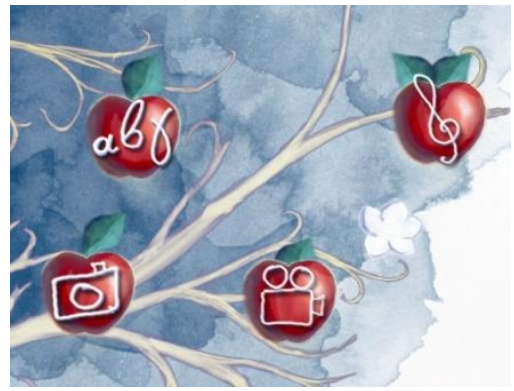

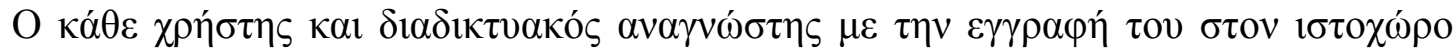

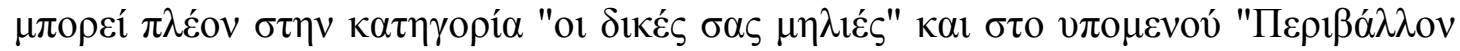

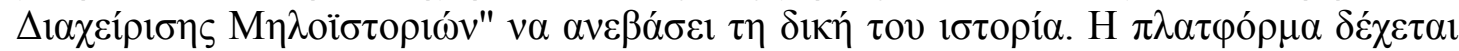

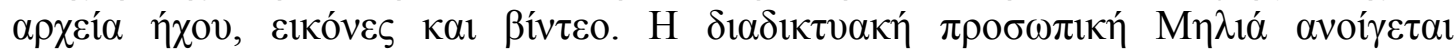

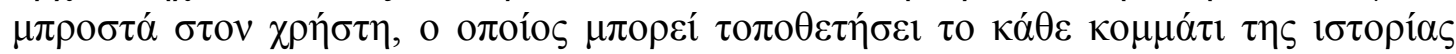

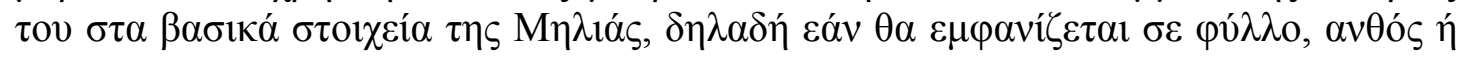

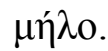

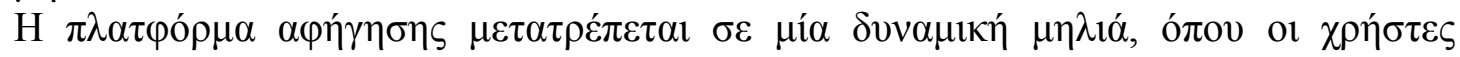

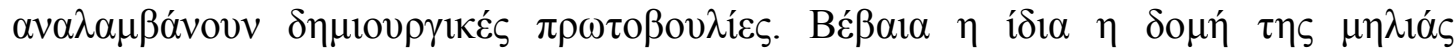

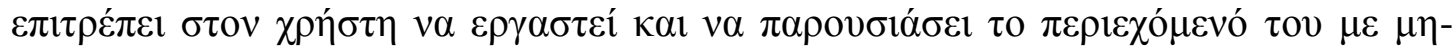

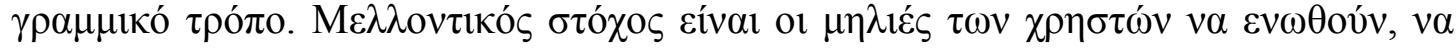

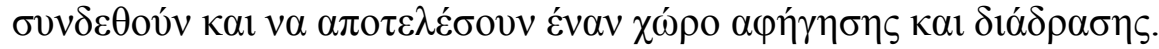

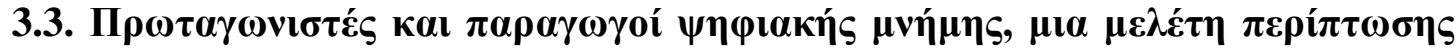

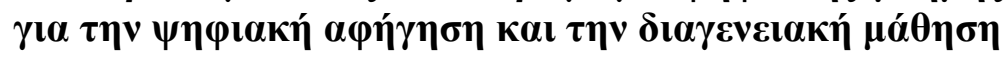

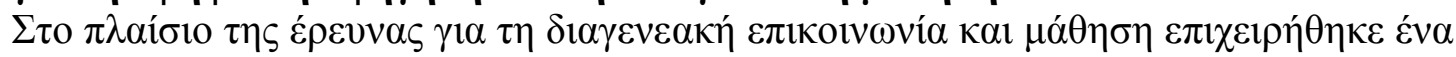

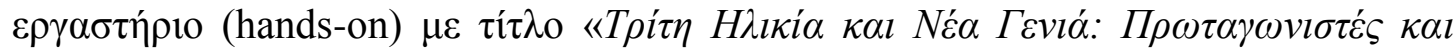

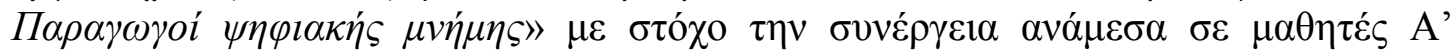

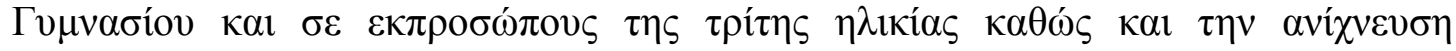

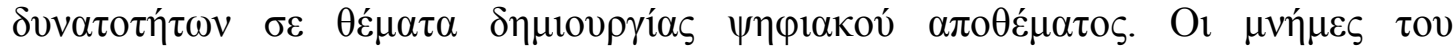

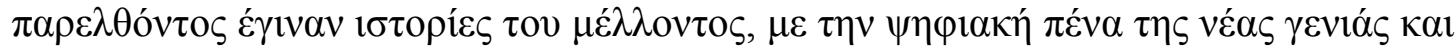

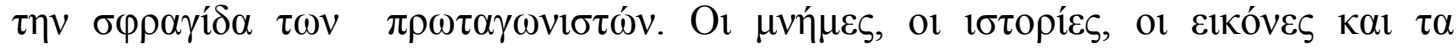




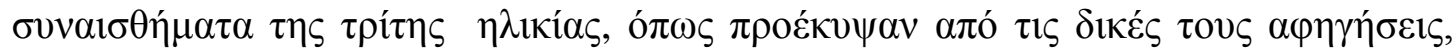

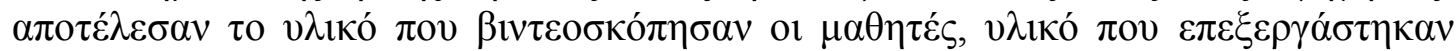

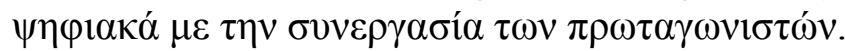

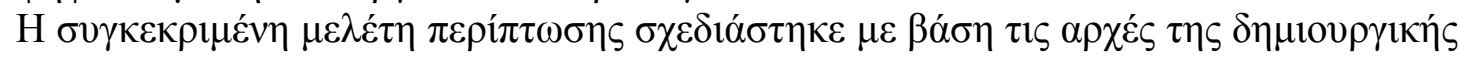

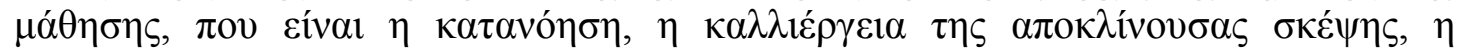

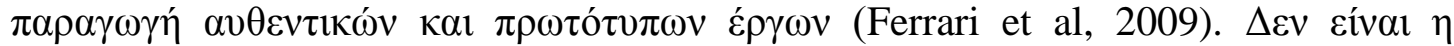

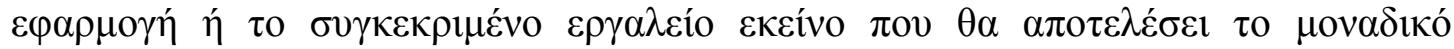

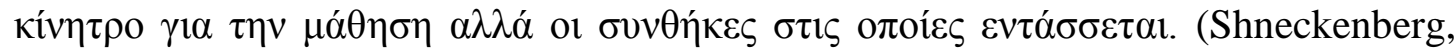

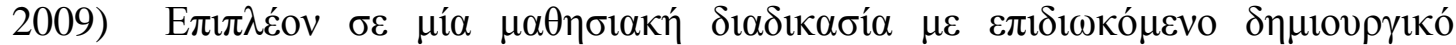

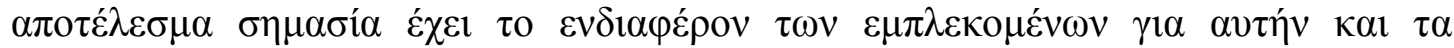

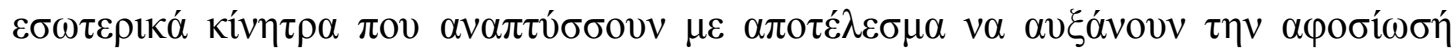

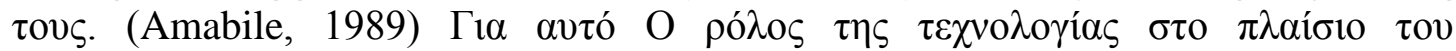

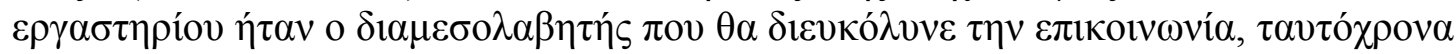

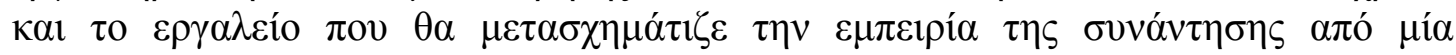

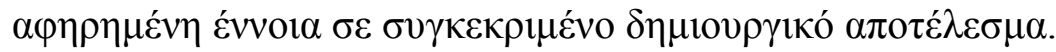

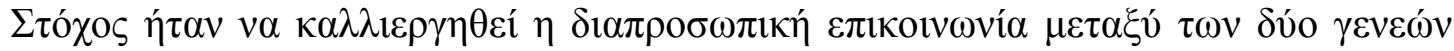

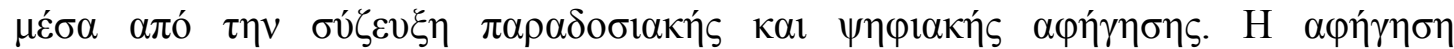

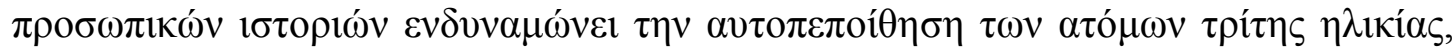

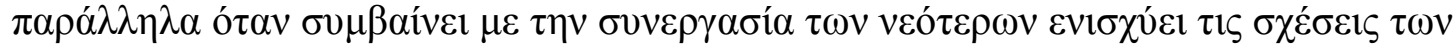

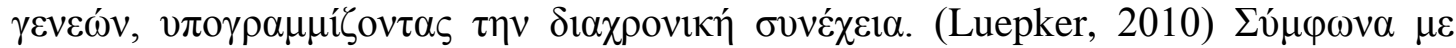

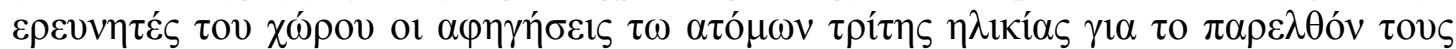

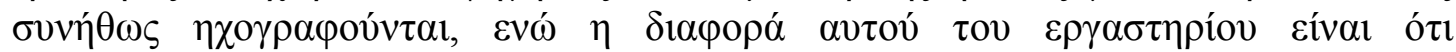

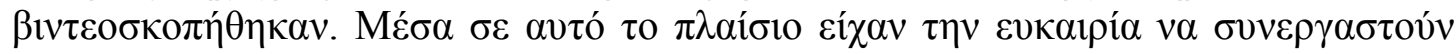

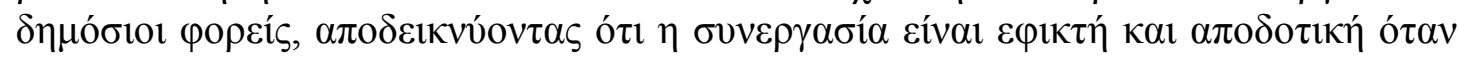

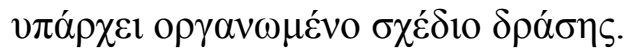

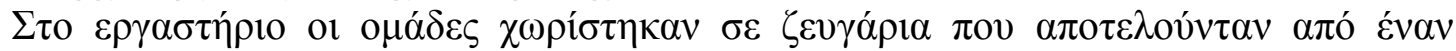

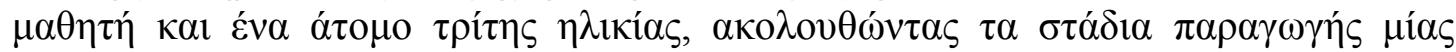

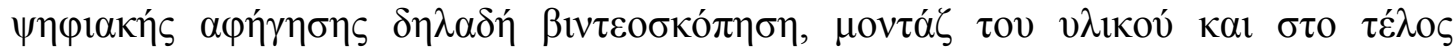

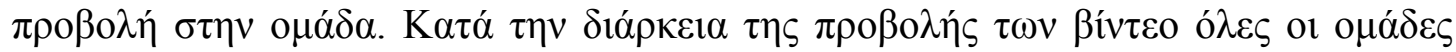

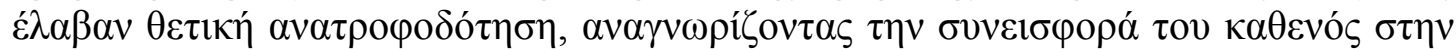

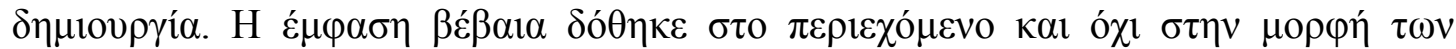

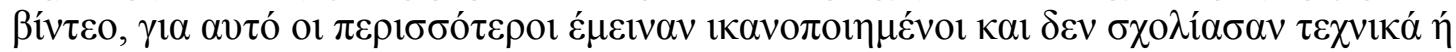

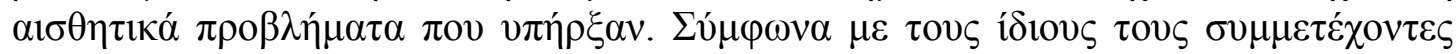

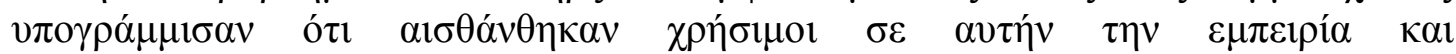

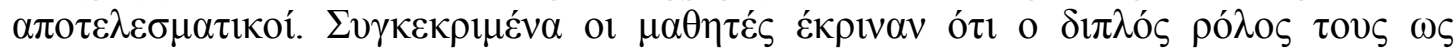

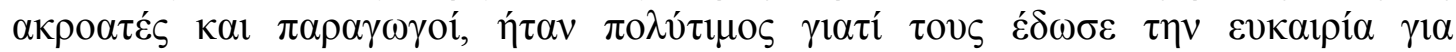

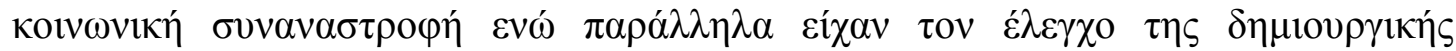

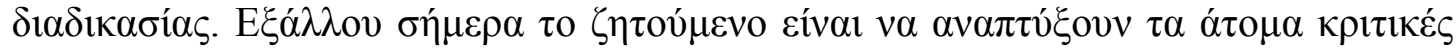

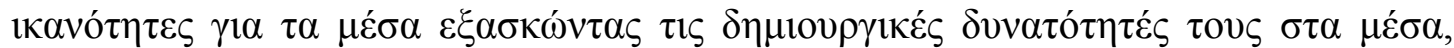

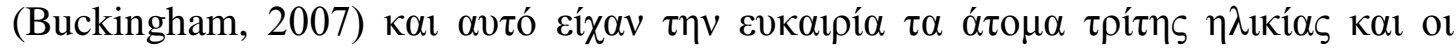

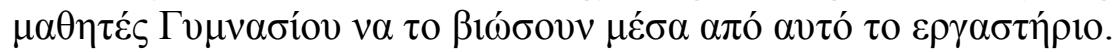

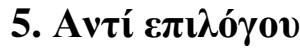

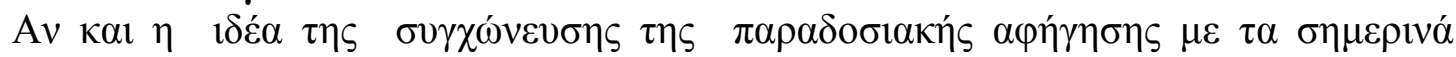

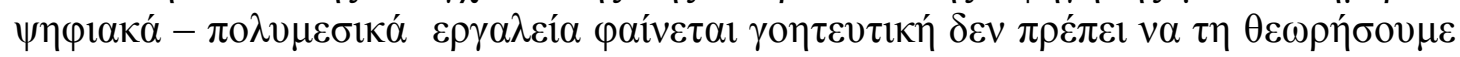

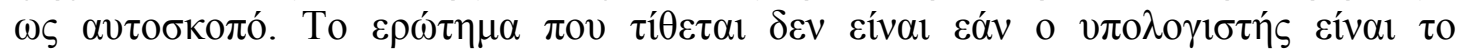

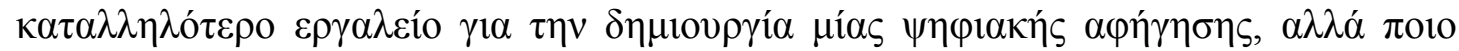

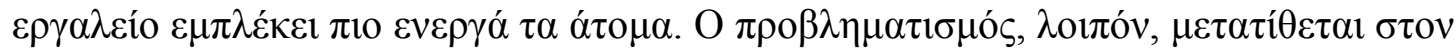

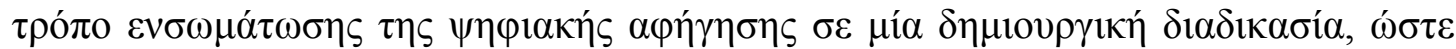

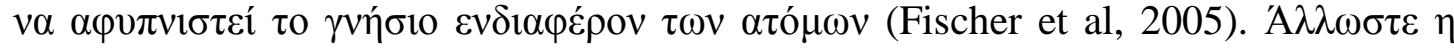




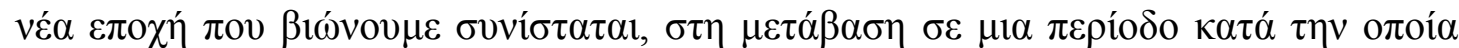

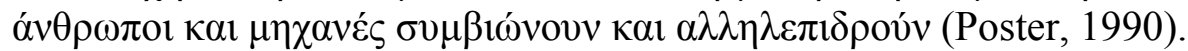

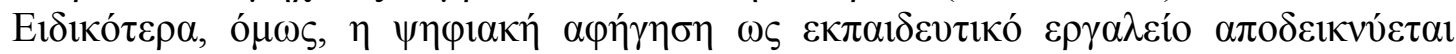

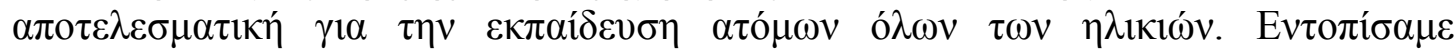

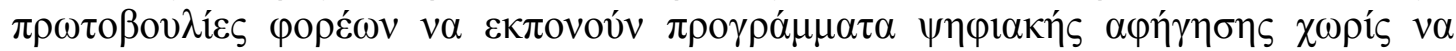

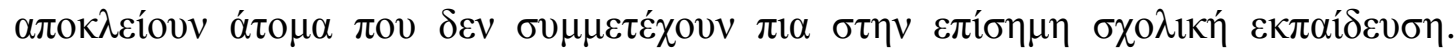

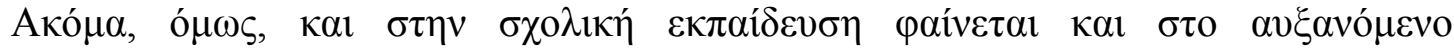

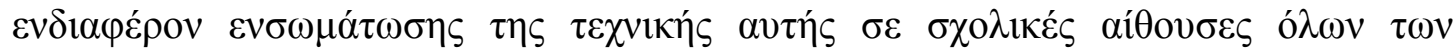
$\beta \alpha \theta \mu i ́ \delta \omega v$.

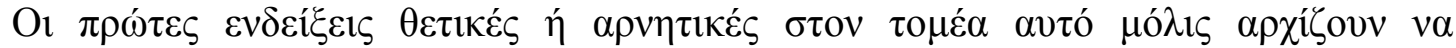

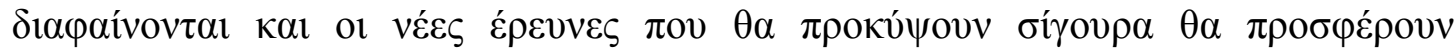

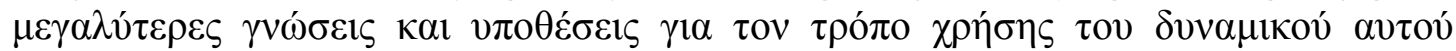

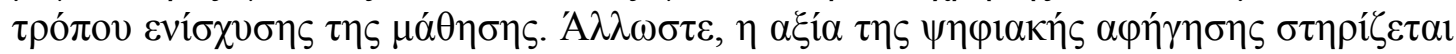

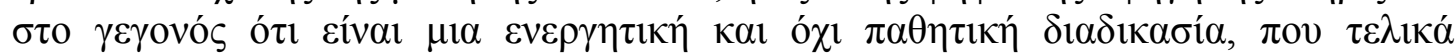

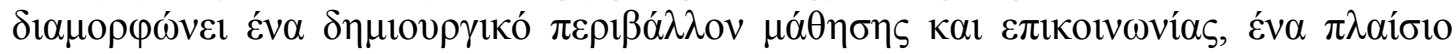

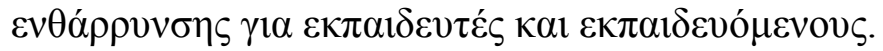

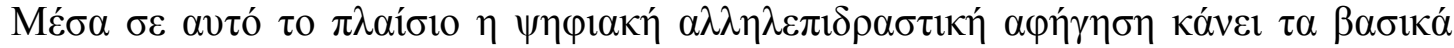

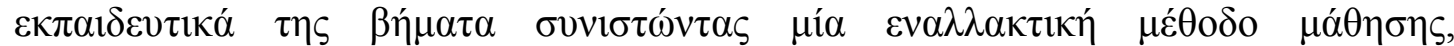

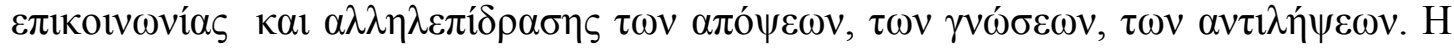

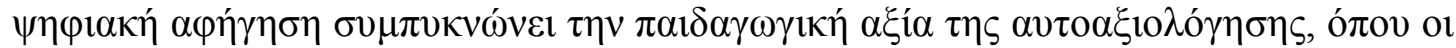

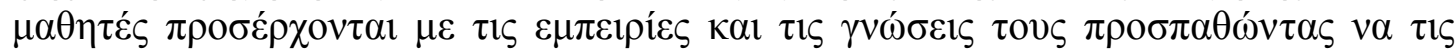

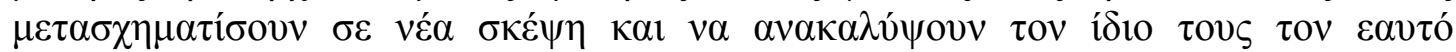

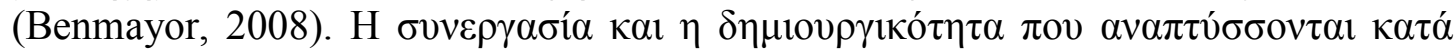

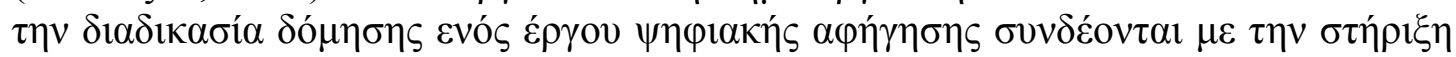

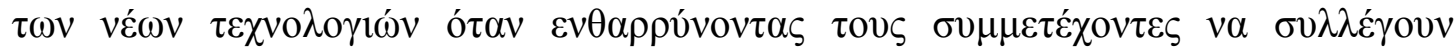

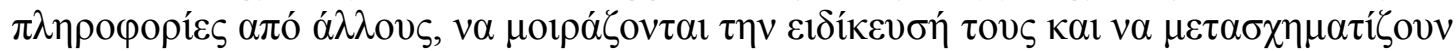

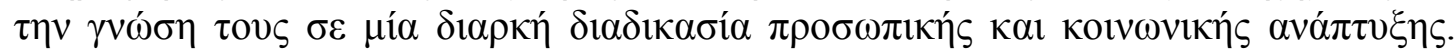

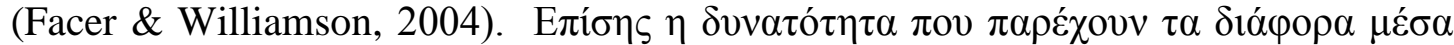

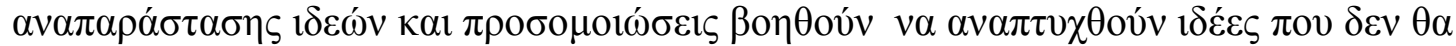

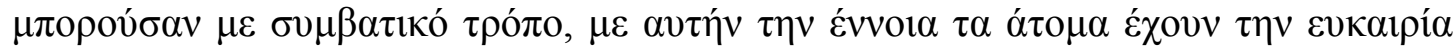

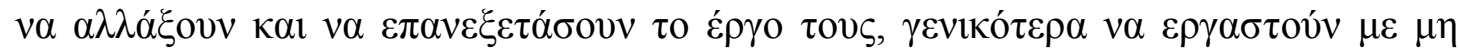

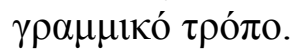

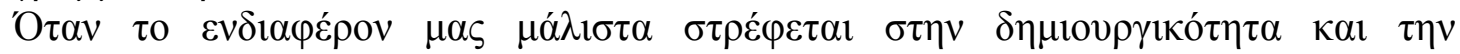

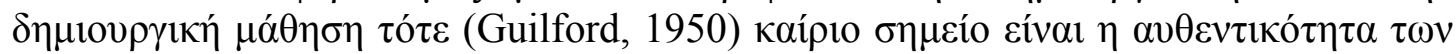

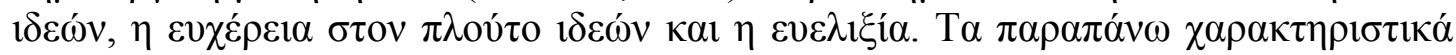

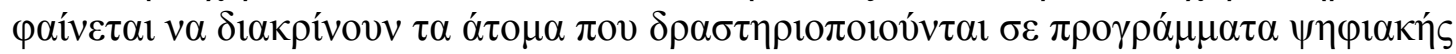

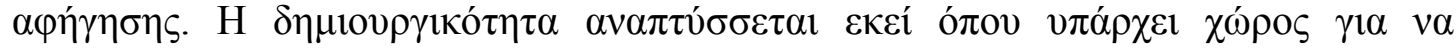

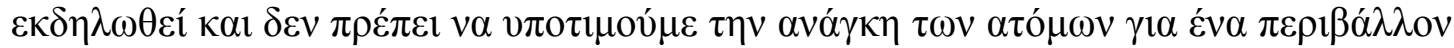

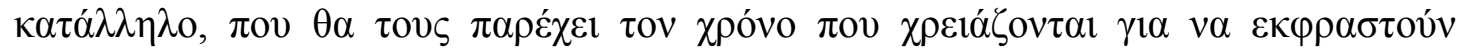

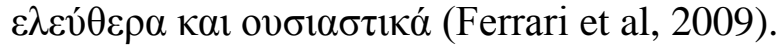

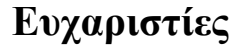

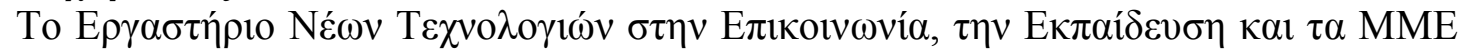

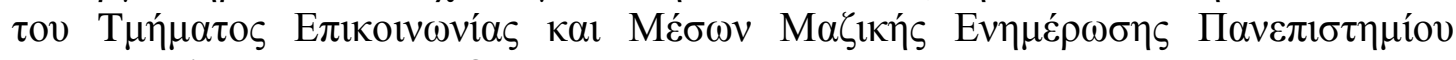

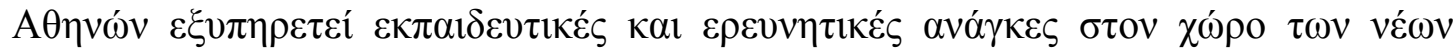

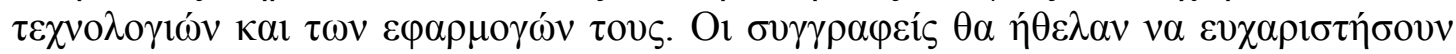

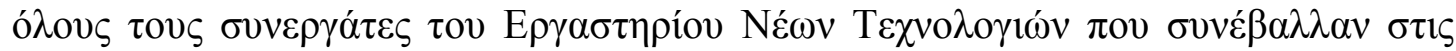

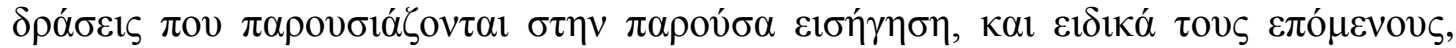

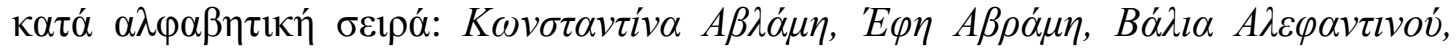

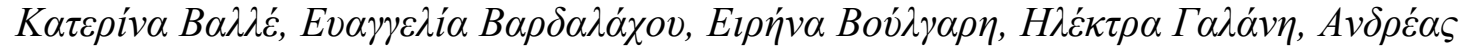




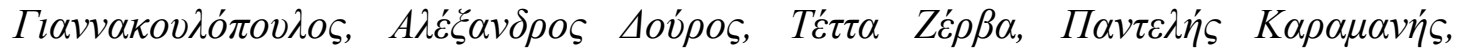

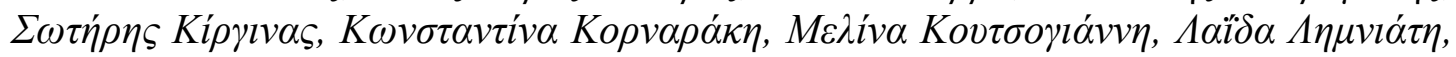

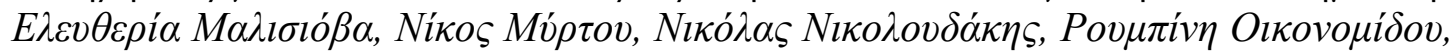

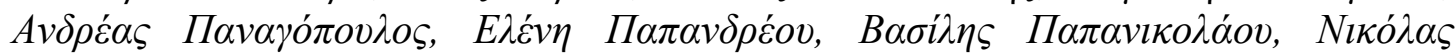

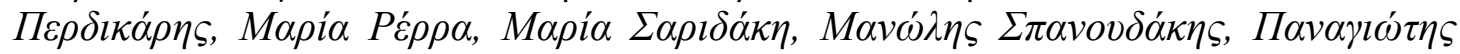

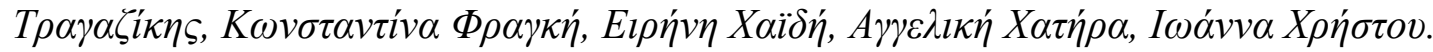

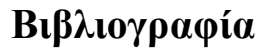

Abram, S., Learning not to answer questions, MultiMedia \& Internet @ Schools, November/December 2007, The pipeline column

Amabile, T.M. (1989). Growing up creative ,New York:Crown Publishing Group,Inc

Barthes, R. (1981). Introduction à l' analyse structural du recit, Seuil

Benmayor, R. (2008). Digital Storytelling as a Signature Pedagogy for the New Humanities, in Arts and Humanities in Higher Education, v.7(2), pp 188-204

Brown, J.S. et al (2005). Storytelling in organizations: why storytelling is transforming 21 st century, US, Butterworth-Heinemann

Buckingham, D. (2002). Children and Media, UNESCO

Buckingham, D. (2007). Media education goes digital: an introduction, Learning, Media and Technology, 32 (2),pp 111-119

Di Blas, N., Garzotto, F. Paolini, P., Sabiescu, A. (2009). Digital Storytelling as a Whole Class Learning Activity: Lessons from a Three-Years Project, in Proceedings of the 2nd Joint international Conference on Interactive Digital Storytelling, Eds. I. A. Iurgel, Zagalo, N., Petta, P., Lecture Notes In Computer Science, vol. 5915, pp 14-29

Ferrari, A., Cachia, R., Punie, Y. (2009). Innovation and Creativity in Education and Training in the EU Member States: Fostering Creative Learning and Supporting Innovative Teaching, Luxembourg, Office for Official Publications of the European Communities

Fischer, G., Giaccardi, E., Eden, H., Sugimoto, M., \& Ye, Y. (2005). Beyond binary choices: Integrating individual and social creativity. International Journal of Human-Computer Studies, 63(4-5), pp. 482-512

Frazel, M., (2010). Digital Storytelling Guide for educators, ISTE, USA

Garzotto, F., Rizzo, F. (2005). Interactive Story Telling, Cooperative e-Learning, and kids: the FaTe2 Field Study, in Proc. IDC'05 - Interaction Design and Children International Conference, Boulder (CO, USA), ACM Press

Gilmor, D. (2006). We the Media, Grassroots journalism for the people by the people. Retrieved on 10 June, 2011 from http://oreilly.com/catalog/wemedia/book/

Guilford, J.P. (1950). Creativity. American Psychologist, vol.5( 9), 444-454

Handler-Miller, C. (2004). Digital Storytelling, Elsevier Science \& Technology

Jenkins, H. (2006). Convergence culture where old and new media collide, New York University Press

Jesper, J. (2001). Games Telling stories? - A brief note on games and narratives, The international journal of computer game research http://theunshaven.rooms.cwal.net/Storage/Readings/Reading\%2007\%20\%20Games\%20Telling\%20Stories\%20\%5BJesper\%20Juul\%5D.PDF

Joyce, M. (1995). Of Two Minds: Hypertext, Pedagogy, and Poetics, Ann Arbor, University of Michigan Press

Lambert, J. (2002). Digital Storytelling: Capturing lives, creating community. Berkeley, CA, Digital Diner Press

Luepker E. T. (2010). Videotaped Life Review: Its Personal and Intergenerational Impact, Clinical Social Work Journal v. 38, pp 183-192

Manovich, L. (2002). The Language of New Media, Cambridge, The MIT Press

McLellan, H. (2006). Digital Storytelling in Higher Education, Journal of Computing in Higher Education, Vol. 19(1), pp. 65-79

Mello, R. (2001). The power of storytelling: How oral narrative influences children's relationships in classrooms. International Journal of Education and the Arts. 2(1).

Ohler, J. (2008). Digital Storytelling in the Classroom. New media pathways to Literacy, Learning and Creativity, Corwin Press

Poster, M. (1990). The Mode of Information, Chicago, The University of Chicago Press

Rideout, V.-Vanderwater, E., Wartella, E. (2003). Zero to six: Electronic media in the Lives of Infants, Toddlers and Preschoolers, CA, Kaiser Family Foundation 
Ryan, M.-L. (2002). Beyond the Myth and Metaphor: Narrative in Digital Media, in Poetics Today, 23 (4), pp. 581-609, Duke University Press

Sandars, J., \& Frith, G. (2009). Reflect 2.0. Using Digital Storytelling to develop reflective learning by the use of Next Generation Technologies and Practices. JISC, pp. 1-5

Schank, R. (1990). Tell me a story: A new look at real and artificial memory. New York, Charles Scribner's Sons

Semali, L., (2003). Ways with Visual Languages: Making the Case for Critical Media Literacy, Clearing House, v.76, no 6, pp. 271-277

Shneckenberg, D. (2009). Understanding the real barriers to technology-enhanced innovation in higher education, Educational Research, Vol.51, No 4, December 2009, pp. 411-424

Snelson, C. \& Sheffield, A. (2009). Digital Storytelling in a Web 2.0 World, TCC, pp. 159- 167

Sobers, S. (2008). Consequences and coincidences: A case study of experimental play in media literacy, Journal of Media Practice, vol. 9, n1, pp53-66

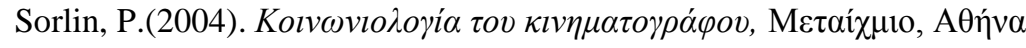

Spieling, U. (2004). Conceptual models for Interactive Storytelling in Knowledge Media Applications, Technologies for Interactive Digital Storytelling and Entertainment, Second International Conference, TIDSE 2004, Darmstadt, Germany, June 24-26, 2004, Proceedings, pp. 171-176, Springer Berlin / Heidelber

Vaucelle, C., Ishii, H. (2009). Play-it-by-eye! Collect movies and improvise perspectives with tangible video objects, in Artificial Intelligence for Engineering Design, Analysis and Manufacturing, Special Issue: Tangible Interaction, v. 23, pp 305-316. Cambridge University Press

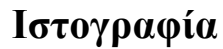

Patient Voices

http://www.patientvoices.org.uk/index.htm

Story Center Berkeley California

http://www.storycenter.org

Capture Wales Project

http://www.bbc.co.uk/wales/arts/yourvideo/queries/capturewales.shtml

Telling Lives, BBC

$\underline{\text { http://www.bbc.co.uk/tellinglives/what.shtml }}$

$\mathrm{M \eta} \lambda i \alpha$

http://www.media.uoa.gr/medialab/milia

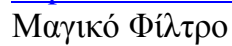

http://www.media.uoa.gr/epinoisi

Mr Binkley's youtube channel

http://www.youtube.com/user/MrBinkley6\#p/a 